\title{
How control and relaxation interventions and virus mutations influence the resurgence of COVID-19
}

\author{
Longbing $\mathrm{Cao}^{1, *}$ and Qing Liu ${ }^{1}$
}

\author{
${ }^{1}$ University of Technology Sydney \\ *longbing.cao@uts.edu.au
}

\section{ABSTRACT}

After a year of the unprecedented COVID-19 pandemic in 2020, the world has been overwhelmed by COVID-19 resurgences in 2021. Resurgences usually cause longer, broader and higher waves of infection, with greater threat to societies and economies compared to first waves. They may be caused by late implementation or early relaxation of non-pharmaceutical interventions (NPIs) such as social restrictions, ineffective interventions, or virus mutations. Here we provide quantitative evidence to characterize epidemiological differences between waves, evaluate possible causes, and predict potential trends under virus mutations. We use an event-driven dynamic epidemiological model embedded with time-dependent intervention interactions to compare two waves of COVID-19 outbreaks, and we quantify the impacts of control or relaxation interventions (called events) on wave patterns. We show the second waves in late 2020 in Germany, France and Italy could have been better contained by either carrying forward the effective interventions from their first waves or implementing better controls and timing. We also obtain the quantitative effects of enforcing or relaxing interventions on various transmissibility levels of coronavirus mutants (like delta or lambda) in the second waves and in the next 30 days. Comprehensive analyses at four levels - vertical (between waves), horizontal (across countries), what-if (scenario simulations on second waves) and future (30-day trend) - in the two 2020 waves in Germany, France and Italy show that (1) intervention fatigue (government and community reluctance to interventions), early relaxations and lagging interventions may be common reasons for the resurgences observed in many countries; (2) timely strong interventions such as full lockdown will contain resurgence; and (3) in the absence of sufficient vaccination, herd immunity and effective antiviral pharmaceutical treatments and with more infectious mutations, the widespread early or fast relaxation of interventions including public activity restrictions will result in a COVID-19 resurgence.

\section{Introduction}

The COVID-19 pandemic has been challenging global society since the SARS-CoV-2 virus was first identified in December 2019 in Wuhan, China. COVID-19 has swept across the world, infecting more than 214 million people and causing over 4 million deaths ${ }^{1}$. The very first waves ${ }^{2}$ in most jurisdictions were successfully managed by immediately and harshly enforcing various non-pharmaceutical interventions (NPIs, for simplicity, we refer 'intervention' to strategies, policies, countermeasures and restrictions to contain the epidemic), such as social distancing, face mask wearing, lockdowns, and travel bans. Our understandings of both the SARS-CoV-2 virus and the COVID-19 disease and the influence of interventions are now much deeper and more comprehensive than in 2020. However, we are still uncertain about the effects of control and relaxation interventions and the increasingly more infectious mutants on the second waves and resurgence of COVID-19. As early as April 2020, there were warnings against the premature relaxation of strict interventions ${ }^{1}$ and the potential risk of a second wave of infections ${ }^{2}$. Many areas that were successful in containing the first wave have suffered from second or multiple waves and hotspot-based resurgence of COVID-19. These areas spread from India, Japan, Singapore and Vietnam in Asia to France, Germany and Italy in Europe, and Brazil in South America. The resurgence transform is further strengthened by rapid SARS-CoV-2 mutations such as the delta and lambda mutants with substantially higher transmissibility and distinct epidemiological attributes. It is vital to deeply understand the causes of COVID-19 resurgence and the different patterns between waves, and evaluate how hard or soft interventions influence resurgence, and how more infectious virus mutations affect resurgence. This work addresses these major issues.

What do we know about COVID-19 resurgence? Resurgence over multiple waves can be illustrated by what happened in some European countries. At the beginning of the first wave in early 2020, most European countries adopted and adjusted a series of strong interventions, which successfully reduced cases and the trajectory of growth. Taking Germany as an example, their cases almost doubled within one day on 1 March 2020. Various interventions were then implemented, including but not limited to cancelling large-scale events, shutting down schools, childcare and businesses, and enforcing restrictions on

\footnotetext{
${ }^{1}$ Accessed on 20 August 2021 at https: / / www . worldometers.info/coronavirus.

${ }^{2}$ Here 'an epidemic wave' has a natural pattern of peaks and valleys of infected cases.
} 

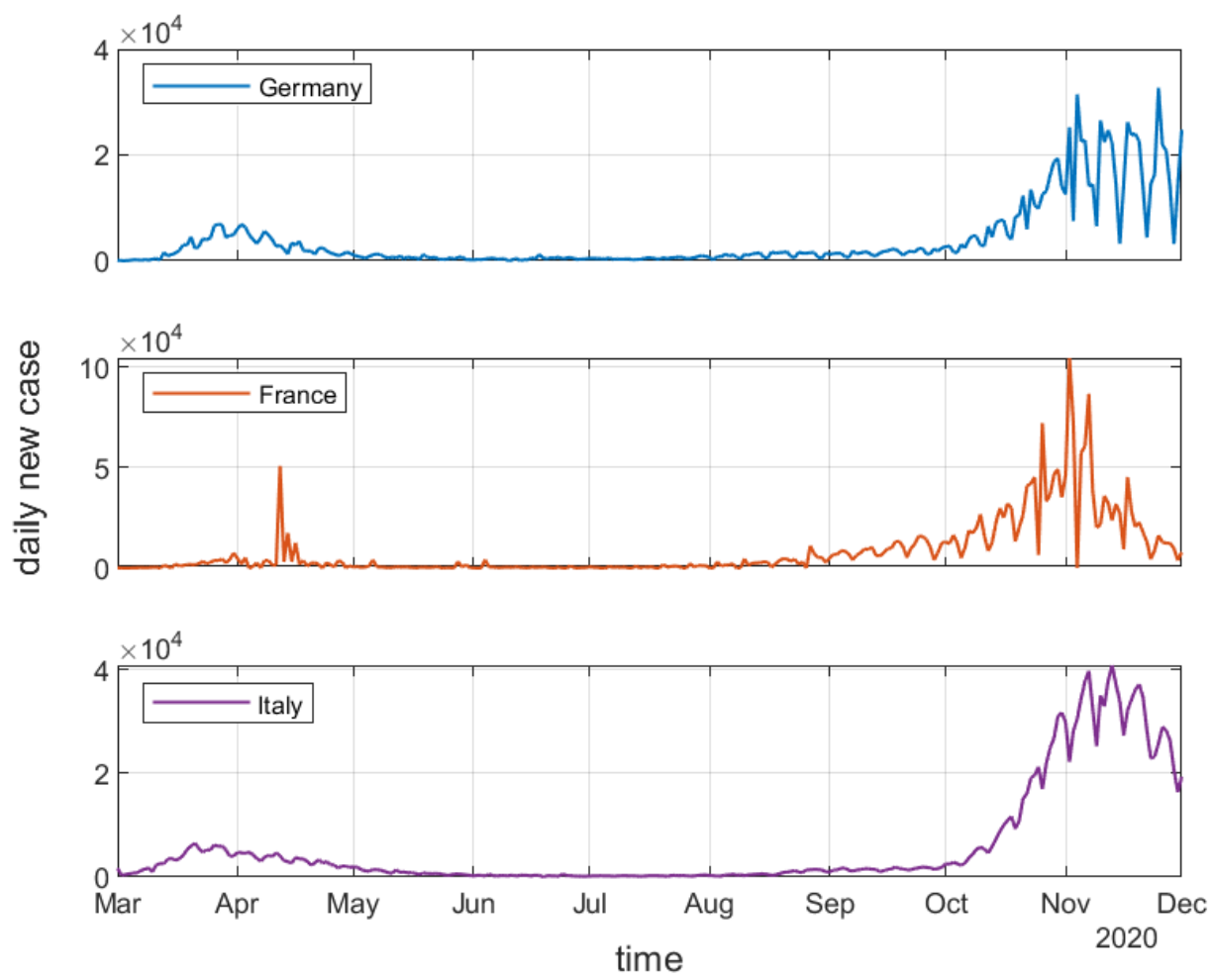

Figure 1. Daily new cases in Germany, France and Italy from March to December 2020. Two evolving waves of COVID-19 epidemic appear in each country, with the three countries having similar but different resurgence attributes.

gathering. Their step-wise increase of these control measures flattened their case curve two months later. However, just a few months later, many European countries had resurgence even worse than their first waves after prematurely or too quickly relaxing their interventions and reopening borders and businesses. As shown in the daily case reports (Figure 1), their second wave peaks were significantly higher than their first waves. For example, in Italy, the peak daily cases reached 6,554 in the first wave, compared with 40,896 cases on 13 November in the second wave, over six times higher than the first peak. Similar differences between waves appeared in most European countries in 2020 and in many other parts of the world in 2021, such as Australia, Vietnam, South Korea and the USA.

There may be various significant and distinct factors driving the COVID-19 resurgence and their differences from the first waves in individual regions, including the more infectious viral mutants. A critical and common trigger is the premature and fast relaxation of interventions, including hygiene behaviors, social and business restrictions, and border control. Despite the positive effect of interventions on flattening the curves, the harsh interventions and the 'new normal' also had significant negative effects on individuals, society and the economy. Enforcing strong interventions such as social distancing and gathering restrictions significantly inconveniences people's daily life. Travel bans, lockdowns and curfews also seriously disrupt social and economic activities, increasing unemployment, poverty and inequality with adverse psychological impacts and pressure on governments. Governments face the dual burden of both providing financial support and being pressured to reopen and recover $^{3}$. Consequently, after months of strict interventions, requests or demands for increasing psychological, behavioral, public and political relaxation emerge over time and spread from one region to another. Subsequently, most countries relaxed their interventions by easing or lifting social distancing restrictions, and reopening schools and workplaces. However, the virus has not been eliminated and even worse mutants have emerged and spread through households, strangers, communities or airborne transmission. The fast relaxation of interventions supports virus transmission. This eventually causes multiple waves and resurgence.

The overwhelming global COVID-19 resurgence and its many uncertainties require in-depth quantification and comparison of wave behaviors and epidemiological features of second waves and resurgence, their differences from first waves, and relationships to external factors in particular interventions and mutations. However, the related research is very limited with focus mainly on (1) describing the epidemiological characteristics of second waves, (2) comparing the epidemiological attributes of first and second waves, and (3) forecasting resurgence including the impact of external factors. Accordingly, first, 
to understand the specific characteristics of the second waves, the dynamics of COVID-19 transmission and outbreak in three European countries has been simulated by the epidemic renormalization group approach $\mathrm{in}^{4}$ or stochastic SEIR model ${ }^{5}$, together with first wave data. The highest infection among young adults was analyzed in ${ }^{6}$ with incidence data by age groups in 25 European countries and studies 7,8 uncovered the reduced case fatality rate from a global and continental view. Second, research also shows that the second wave has different characteristics from the first although both waves share similar transmission mechanisms. Investigating the severity and characteristics of hospitalized patients in the two waves in Reus in Spain ${ }^{9}$ found that patients in the second wave were younger and their hospitalization duration and case fatality rate were lower than in the first wave. Similar conclusions were drawn in Japan ${ }^{10}$. In Italy, the effects of the two waves on public health and their impact trends, fatality rates, admissions to ICUs and confirmed cases were shown to be statistically different ${ }^{11}$. Lastly, several studies have predicted the occurrences of second waves or COVID-19 resurgence associated with external factors such as easing interventions ${ }^{12-14}$ or intervention fatigue (government and community reluctance to interventions) ${ }^{15}$, regrowth patterns under different (e.g., mobility) scenarios ${ }^{16}$, estimated the risk or trend of resurgence ${ }^{17-20}$ and the impact of integrating physical distancing and vaccination ${ }^{21}$, quarantine, testing and contact tracing ${ }^{22}$, non-pharmaceutical public health intervention ${ }^{23}$, social distancing and loss of immunity ${ }^{24}$, virus mutation ${ }^{25}$ and air pollution ${ }^{26}$ on future waves, and urged prompt and effective actions to prevent COVID-19 resurgence. By forecasting second waves based on behavior disease interactions, it is believed that the second waves are related to the interactions between disease dynamics and social processes ${ }^{27}$.

The problem and findings. The existing studies mainly focus on specific aspects and a qualitative to descriptive analysis of second waves. The contrasting epidemiological characteristics between waves remain largely unknown. Epidemiological predictive analysis of multi-waves has received limited attention, leaving significant gaps in areas including deep epidemiological characterization and simulation of the resurgence and deep insight into how various interventions affect the resurgence. In reality, the later waves become more global, repetitive, infectious and variable, particularly with the evolving mutants. There are many important questions to be answered by evidence. For example, what leads to the second waves? Why are later waves more severe (e.g., in terms of cases and impacts) than the first? Is the knowledge learned from the first wave transferable to better control later waves? How does virus mutation affect the epidemic?

In this research, we systematically quantify the interactions between people at different epidemic states, epidemic processes, intervention strategies, public activities and virus mutations and their influences on COVID-19 wave differences and resurgence. To this end, we involve epidemiological knowledge, data-driven discovery, dynamic interaction and event analysis. Evidence and findings are extracted from domain, data and event-driven dynamic interaction modeling of COVID-19 first-to-second wave cases, interventions, social activities and virus mutations to address several fundamental questions about COVID-19 resurgence: (1) How do epidemiological attributes change over the first to second wave in different countries? (2) How does different severity, number and timing of interventions individually and cumulatively affect the behaviors and trends of two waves? (3) How would different control and relaxation intervention strategies influence the next 30-day trends following the second waves? (4) How would more infectious virus mutations influence the second waves and the next 30-day trends under strong and weak interventions?

To answer question (1), we quantify the epidemiological attributes of the first and second waves and analyze the different behaviors and patterns between waves in Germany, France and Italy. We obtain quantitative and predictive evidence on the difference between waves with case studies in Germany, France and Italy. With question (2), we characterize the dynamic processes of the first-to-second wave evolution and their sensitivity to different severity, number and timing of interventions and social activities implemented in the two waves. We thus infer the individual and cumulative impact of control and relaxation interventions, early and late interventions, and more and less interventions implemented in the two waves on containing two waves. To address question (3), we simulate six scenarios of tightening or relaxing interventions and social activities and predict and compare their effects on the daily new cases, active cases, and total confirmed cases in the next 30 days following the second waves. These scenarios reflect different response strategies and behaviors by governments, individuals and societies to the second waves, such as enforcing interventions or relaxing interventions and restrictions on socioeconomic activities to different extents. Lastly, with question (4), we first simulate and evaluate the influence of more infectious virus mutants (such as delta and lambda) with different levels of transmissibility on the second waves when the actual strong and weak interventions are carried from their first and second waves. Then, we predict the next 30-day case trends following the second wave if different levels of transmissibility of virus mutants appeared.

Accordingly, we develop a dynamic event-driven generalized SEIR model SPEIQRD. Here, events broadly refer to any control or relaxation interventions implemented by governments, individuals or communities that may drive the dynamic change of COVID-19 population states and coverage and influence the epidemic processes, transmissions and trends. Taking these external factors into consideration, SPEIQRD models epidemiological states, state transition and attributes of population and epidemic processes by involving their interactions with and the influence of external dynamic events. We apply the model in the first and second waves in 2020 in Germany, France and Italy as a case study to extract evidence on the above questions. We conduct comprehensive quantitative analyses at four levels - vertical between waves in each country, horizontal across countries, 
what-if with simulations on the second waves, and future for the next 10 and 30-day trends. We further estimate, simulate, predict and evaluate the above approaches to each question, including (1) the different epidemiological attributes, behaviors and patterns of first and second waves between countries; (2) the individual and cumulative impact of control, relaxation, early and late interventions on wave patterns; (3) the effects of tightening or relaxing interventions in the first-to-second wave evolution, the second waves, and next 30-day trends; (4) the influence of changing the virus transimissibility on the second waves and 30-day trends under strong or weak interventions.

These analyses obtain comprehensive evidence, insights and results about the dynamics, evolution, attributes and causes of COVID-19 resurgence and the influence of external events and virus mutations on the resurgence. First, compared to the first waves, second waves show obviously different epidemiological attributes including more infected cases, and longer incubation periods but lower quarantine rates. These differences result from intervention fatigue, the relaxed and late implementation of interventions, the lagging control measures, and higher deconfinement activities ${ }^{317}$, which lead to more protected individuals rejoining the susceptible population. Second, different levels of interventions have varied effects. Early and strong control interventions push down cases quickly. It is essential to enforce tougher containment earlier in severe epidemic situations. Third, too late implementation of interventions or too early reopening likely results in an exponential increase of daily new cases, more serious transmissions, longer incubation and harder, late but longer interventions to get the epidemic under control. Fourth, with more infectious virus mutants, tightening or relaxing interventions would lead to contrasting results of infection, indicating the need for instant implementation of effective interventions at the initial outbreak of a highly infectious mutation. Lastly, different effects and trends of second waves and next 30 days occur under different strategies: maintaining, strengthening or relaxing the interventions; different severity, number and timing of interventions; and under various levels of infectious mutations. We determine the influence of enforcing and relaxing interventions on their wave differences; the better containment of their second waves when more effective interventions were carried forward from their first waves; and the higher risk of infection if more infectious virus mutants are not well controlled.

This research significantly complements the existing work that focuses on either specific epidemiological attributes or the second wave itself. Our results provide deep evidence, explanations and insights about important questions and objectives of understanding COVID-19 resurgence, multi-wave evolution and wave differences, the interactions between interventions and epidemic dynamics, the effect of various interventions on resurgences, and appropriate intervention strategies on containing virus mutations. The findings and analyses in the following sections provide evidence for governments and policymakers to systematically manage the severity, number and timing of interventions on resurgences; appropriately balance case development scenarios and intervention strategies; and effectively predict and intervene future COVID-19 resurgence. The research also shows that governments and the public have to be prepared for the effects of inappropriate containment and inappropriate timing in managing resurgence and mutations. The research is also useful in the increasing debate on whether elimination (zero COVID, such as the practices undertaken in countries like China), eradication or suppression ("living with the virus" at an acceptable level, like the approaches undertaken in countries like the UK and US) and when full reopening are reasonable in practice.

\section{Methods}

Data. For the purpose of this research, we choose the first and second waves in Germany, France and Italy in 2020 as case studies. They clearly showed the first-to-second wave evolution with clear wave differences and different intervention strategies. We can collect the ground truth information of daily cases and sequentially interventions and public activities to verify and explain the our modeling objectives and results. Both COVID-19 case data and NPI-related event information are used. We use the JHU CSSE COVID-19 data ${ }^{28}$, which records the worldwide daily case reports including total confirmed cases, recovered cases, and deaths. The event information includes both government-initiated mitigation policies and public activities, which influence COVID-19 transmission and resurgence. It is collected from Wikipedia for the COVID-19 pandemic in Germany ${ }^{4}$, the COVID-19 pandemic in France ${ }^{5}$, and the COVID-19 pandemic in Italy ${ }^{6}$. The OxCGRT (the Oxford COVID-19 Government Response Tracker) also collects systematic information on government responses to COVID-19 across countries in four categories: containment and closure, economic response, health systems, and miscellaneous ${ }^{29}$. However, the policy information recorded in this dataset is somewhat inconsistent and less timely than the events reported above. Moreover, large public events that may influence the COVID-19 trend in a country are excluded from OxCGRT. We therefore collect event information from the Wikipedia sources.

As shown in Tables 1-3, the extracted major events from the beginning of March 2020 to the end of November 2020 are classified into two types: $\mathrm{C}$ for control policies, measures and actions; and $\mathrm{R}$ for lifting or relaxing restrictions. During this

\footnotetext{
${ }^{3}$ https://www.who.int/publications/i/item/wHO-2019-nCoV-IHR-Quarantine-2021.1

${ }^{4}$ https://en.wikipedia.org/wiki/COVID-19_pandemic_in_Germany

${ }^{5}$ https://en.wikipedia.org/wiki/COVID-19_pandemic_in_France

${ }^{6}$ https://en.wikipedia.org/wiki/COVID-19_pandemic_in_Italy
} 
Table 1. The major interventions undertaken during the two COVID-19 waves in Germany.

\begin{tabular}{|c|l|c|}
\hline Date & \multicolumn{1}{|c|}{ Event } & Type \\
\hline 08 Mar. & cancel public events of more than 1,000 attendees & $\mathrm{C}$ \\
13 Mar. & close schools and nurseries & $\mathrm{C}$ \\
16 Mar. & implement almost shutdown & $\mathrm{C}$ \\
22 Mar. & forbid gatherings & $\mathrm{C}$ \\
31 Mar. & encourage mask wearing & $\mathrm{C}$ \\
20 Apr. & start to reopen shops & $\mathrm{R}$ \\
30 Apr. & expand reopening under strict social distancing conditions & $\mathrm{R}$ \\
15 May & ease border controls & $\mathrm{R}$ \\
03 Jun. & allow travel to all 26 EU countries & $\mathrm{R}$ \\
13 Jun. & lift most contact restrictions & $\mathrm{R}$ \\
01 Aug. & have a 20,000 people protest ignoring mask-wearing and physical & $\mathrm{R}$ \\
& distancing requirements & \\
20 Sep. & have a protest with mask-wearing requirements & $\mathrm{R}$ \\
17 Oct. & start staying at home and contact tracing & $\mathrm{C}$ \\
02 Nov. & start partial lockdown & $\mathrm{C}$ \\
25 Nov. & extend partial lockdown, etc. & $\mathrm{C}$ \\
\hline
\end{tabular}

time period, the target countries experienced different first and second waves of COVID-19. Their governments implemented different control and relaxation policies in response to the first and second outbreaks over different stages of the waves and their case movements. Their policies changed from the initial control measures in the first wave to relaxation after the first wave was contained and then to control measures again to contain their COVID-19 resurgence.

Model. The SPEIQRD model is a dynamic event-driven epidemiological compartmental model incorporating epidemiological attributes, intervention impact (event attributes), and their interactions, as shown in Figure 2. Our model not only characterizes the dynamic epidemiological attributes of the COVID-19 pandemic, as by other compartment-based SEIR structures $^{17,30}$, but also quantifies the different impacts of control and relaxation events, differentiates the epidemiological and event attributes over the first-to-second COVID-19 wave evolution, and makes these attributes adjustable for what-if analysis and simulation of complex and different intervention strategies, various transmissibility levels of virus mutations, and their joint effects on wave dynamics and first-to-second wave transform as well as COVID-19 resurgence. This is critical to connect mathematical compartmental models to their external driving factors such as government and public responses, characterize how interventions affect the dynamic transfer from the first to second waves, and how control or relaxation interventions affect protection and deconfinement and eventually the epidemic dynamics individually and cumulatively in complex conditions such as with contrasting intervention strategies and more infectious virus mutations.

First, as an epidemiological model, it partitions the entire population into seven compartments, as shown in Figure 2, to characterize seven unique states of population involved in an epidemic and their state transition over evolving COVID-19 epidemic. The population flow during the epidemic transmission at time $t$ is characterized by Equations (1)-(7), where $S$ is the susceptible population, $E$ is the exposed population, $I$ is the actual infected case number which contains unreported cases, $Q$ is the quarantined case number, $R$ is the recovered number, $D$ is the death number, $P$ is the protected and confined population, and $N$ is the total population. The protected or confined population in the compartment $P$ refers to those who are not susceptible to the disease due to their good self-protection habits or staying away from infectious people. Additionally, we suppose $N$ is a closed population and ignore the natural birth and death numbers as they are negligible compared to the large target population in $N$.

Second, during the pandemic evolution, governments enforce control measures to flatten the curves during outbreaks or relax restrictions to restore socioeconomic activities when infections substantially decline or diminish. To model such different stages of enforcing or relaxing interventions and their effects, SPEIQRD incorporates control and relaxation interventions into the epidemic transmission processes and models their influences by an event-driven dynamic model. An event refers to either an intervention that is undertaken to control the COVID-19 pandemic (or unwind it) or a public activity such as a protest or a public gathering that has a great influence on the trend of COVID-19 cases. Specifically, control interventions are the COVID-19 countermeasures implemented during infection spread and outbreak; relaxation events are policies and activities implemented during the social recovery period to relax or unwind the controls. Besides these interventions, public activities such as large-scale gatherings happening during outbreaks may also influence the COVID-19 curves $^{31}$. For example, in Germany, two large protests on 1 August and 20 September 2020 in which a majority of the participants ignored the mask-wearing and social-distancing requirements likely contributed to the dramatic increase of COVID-19 cases after a few days. Such maskless 
Table 2. The major interventions undertaken during the two COVID-19 waves in France.

\begin{tabular}{|c|l|c|}
\hline Date & \multicolumn{1}{|c|}{ Event } & Type \\
\hline 05 Mar. & ban on gatherings of more than 5,000 people & $\mathrm{C}$ \\
10 Mar. & ban on gatherings of more than 1,000 people & $\mathrm{C}$ \\
14 Mar. & ban on gatherings of more than 100 people & $\mathrm{C}$ \\
16 Mar. & close schools and public establishments & $\mathrm{C}$ \\
17 Mar. & start lockdown & $\mathrm{C}$ \\
12 May & allow business reopening and gatherings per social distancing rules & $\mathrm{R}$ \\
30 May & reopen parks & $\mathrm{R}$ \\
02 Jun. & reopen restaurants and museums & $\mathrm{R}$ \\
15 Jun. & permit demonstrations per social distancing guidelines & $\mathrm{R}$ \\
22 Jun. & reopen cinemas & $\mathrm{R}$ \\
11 Jul. & lift majority of restrictions & $\mathrm{R}$ \\
01 Sep. & require mask wearing in workplaces & $\mathrm{C}$ \\
17 Oct. & start gathering restriction & $\mathrm{C}$ \\
24 Oct. & start overnight curfews & $\mathrm{C}$ \\
30 Oct. & start second national lockdown & $\mathrm{C}$ \\
28 Nov. & reopen non-essential services & $\mathrm{R}$ \\
\hline
\end{tabular}

Table 3. The major interventions undertaken during the two COVID-19 waves in Italy.

\begin{tabular}{|c|l|c|}
\hline Date & \multicolumn{1}{|c|}{ Event } & Type \\
\hline 01 Mar. & start local lockdown & $\mathrm{C}$ \\
04 Mar. & close schools and universities nationwide & $\mathrm{C}$ \\
09 Mar. & start full lockdown & $\mathrm{C}$ \\
11 Mar. & tighten lockdown & $\mathrm{C}$ \\
20 Mar. & implement tighter regulations on free movement & $\mathrm{C}$ \\
24 Mar. & start higher fines for the violation of restrictive measures & $\mathrm{C}$ \\
10 Apr. & reopen some businesses & $\mathrm{R}$ \\
04 May & lift movement restriction and reopen industry & $\mathrm{R}$ \\
18 May. & lift lockdown partially & $\mathrm{R}$ \\
03 Jun. & restore free movement nationwide & $\mathrm{R}$ \\
07 Oct. & impose the use of masks outdoors & $\mathrm{C}$ \\
13 Oct. & forbid gathering and tighten containment & $\mathrm{C}$ \\
15 Oct. & close schools & $\mathrm{C}$ \\
23 Oct. & start regional lockdowns & $\mathrm{C}$ \\
25 Oct. & close restaurants, bars and gyms, etc. & $\mathrm{C}$ \\
\hline
\end{tabular}




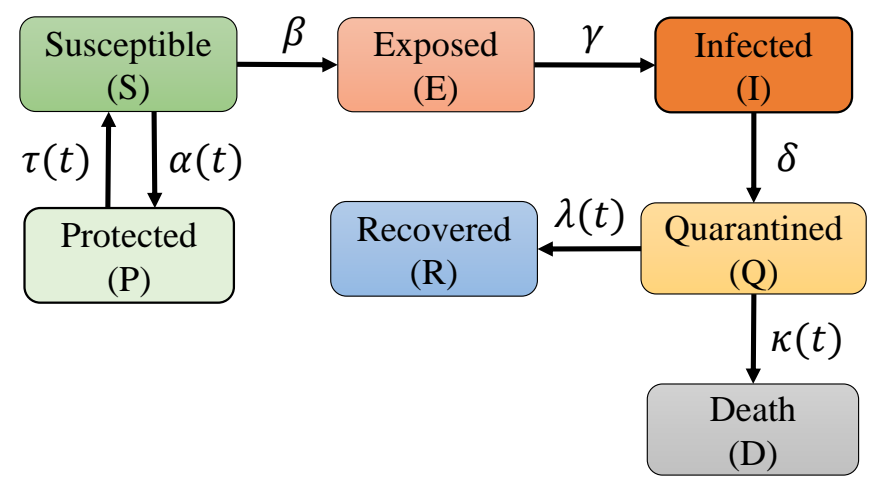

Figure 2. The dynamic event-driven compartmental model SPEIQRD for modeling both epidemiological attributes and intervention impact in the first-to-second wave transfer. Event impact attributes: the protection rate $\alpha$, and the deconfinement rate $\tau$; and epidemiological attributes: the infection rate $\beta$, the incubation rate $\gamma$, the quarantine rate $\delta$, the recovery rate $\lambda$, and the mortality rate $\kappa . \alpha, \tau, \lambda$ and $\kappa$ are time-varying parameters.

gatherings could be an important contributor of COVID-19 resurgence even though it is challenging to infer their specific influence directly. We thus involve them as relaxation events in the model.

SPEIQRD involves the following parameters: the infection rate $\beta$, the incubation rate $\gamma$, the quarantine rate $\delta$, the recovery rate $\lambda$, the mortality rate $\kappa$, the protection rate $\alpha$, and the deconfinement rate $\tau$. $\beta, \gamma, \lambda$ and $\kappa$ measure the epidemiological attributes of the pandemic, $\delta$ measures the quarantine effect, and the parameters $\alpha$ and $\tau$ further characterize the influence of control and relaxation events on the epidemic. A positive protection rate of $\alpha$ models the improvement of public health and self-protection awareness, such as encouraging wearing face masks and good personal hygiene (e.g., hand sanitization). Similarly, a positive deconfinement rate of $\tau$ simulates the return of those people from the confined compartment to the susceptible compartment due to their potential loss of acquired immunity, the deconfinement measures (e.g., society reopening), or other unprotected activities (e.g., large-scale gatherings without mask wearing).

$$
\begin{aligned}
\frac{d S(t)}{d t} & =-\beta \frac{S(t) I(t)}{N}-\alpha(t) S(t)+\tau(t) P(t) \\
\frac{d E(t)}{d t} & =\beta \frac{S(t) I(t)}{N}-\gamma E(t) \\
\frac{d I(t)}{d t} & =\gamma E(t)-\delta I(t) \\
\frac{d Q(t)}{d t} & =\delta I(t)-(\lambda(t)+\kappa(t)) Q(t) \\
\frac{d R(t)}{d t} & =\lambda(t) Q(t) \\
\frac{d D(t)}{d t} & =\kappa(t) Q(t) \\
\frac{d P(t)}{d t} & =\alpha(t) S(t)-\tau(t) P(t)
\end{aligned}
$$

Further, epidemic dynamics are captured by the time-varying parameters: the recovery rate $\lambda$, the mortality rate $\kappa$, the protection rate $\alpha$, and the deconfinement rate $\tau$. These parameters measure both dynamic states of populations in the epidemic transmission and the sequential control and relaxation interventions and their evolving impact on the epidemic transformation over time. This captures the interactions between epidemiological attributes and intervention impact attributes. As shown by the case movements in Figures (3)-(5), the recovered and death populations evolve over time, i.e., described by the time-dependent cure and mortality rates as explored in ${ }^{17,30,32}$. The estimated recovered and death rates are not constant in both the first (which are complete) and second (which are partial and still evolve at that time) waves, except the recovery rate of the second wave in France.

In addition, to capture the different trends of recovered and death cases affected by the interventions, quarantine and protection, we simulate the recovery rate $\lambda$ and the mortality rate $\kappa$ in terms of three optional exponential functions respectively 
in Equations (8)-(9).

$$
\begin{gathered}
\lambda(t)= \begin{cases}\lambda_{0}+e^{-\lambda_{1}\left(t+\lambda_{2}\right)}, & \text { or } \\
\lambda_{0}\left(1+e^{\lambda_{1}\left(t-\lambda_{2}\right)}\right), & \text { or } \\
\frac{\lambda_{0}}{1+e^{-\lambda_{1}\left(t-\lambda_{2}\right)}}\end{cases} \\
\kappa(t)= \begin{cases}\kappa_{0}+e^{-\kappa_{1}\left(t+\kappa_{2}\right)}, & \text { or } \\
\kappa_{0}\left(e^{-\left(\kappa_{1}\left(t-\kappa_{2}\right)\right)^{2}}\right), & \text { or } \\
\frac{\kappa_{0}}{e^{\kappa_{1}\left(t-\kappa_{2}\right)}+e^{-\kappa_{1}\left(t-\kappa_{2}\right)}} & \end{cases}
\end{gathered}
$$

Specifically, unlike studies that treat the infection rate as time-dependent ${ }^{33,34}$, we consider it constant since this may better capture the intrinsic epidemiological attribute of the virus in a fixed period; the infection ability of the virus may remain stable when interventions are enforced to protect people and contain transmission. On the other hand, the dynamics of infection rate observable from the daily cases is sensitive to the enforcement and adjustment of control or relaxation policies and people's increasing self-protection awareness and response to the epidemic. To capture the influence of these factors on infection, we introduce the protected compartment for those people under protection during transmission and further measure the influence of enforcing or relaxing interventions on these people in terms of dynamic protection and deconfinement rates.

To quantify the event effects on COVID-19 transmissions, both the protection rate $\alpha$ and the deconfinement rate $\tau$ are assumed to be event-driven and follow step functions shown in Equations (10)-(11), where $m$ refers to the total number of events, and $\rho$ and $\varphi$ are indicators for control events (i.e., type $\mathrm{C}$ in Tables 1-3) and relaxation events (i.e., type $\mathrm{R}$ in Tables 1-3), respectively. $\pi$ represents the impact level of a control or relaxation intervention (or a public activity). Specifically, $\rho_{n, t}$ equals 1 if the $n^{\text {th }}$ event is a control measure and in place at time $t$; otherwise it equals 0 . Similarly, $\varphi_{n, t}$ equals 1 if the $n^{\text {th }}$ event is a relaxation policy and in place at time $t$; otherwise it equals 0 . When control measures are ongoing, more people will be protected from being infected under the stricter measures, and their self-protection awareness is also enhanced, correspondingly increasing the protection rate $\alpha$, as shown in Equation 10. However, to revive social and economic activities, governments may relax restrictions progressively, resulting in more people moving back to the susceptible compartment since the virus is still valid, i.e., increasing the deconfinement rate $\tau$ as shown in Equation 11. Large-scale public activities such as protests may violate control measures to convert some protected individuals to susceptible, which is also shown in our design and experiments. Since there is a time delay between an event being undertaken and its actual effect being validated, we introduce the time delay parameter $v$ as an integer on a daily basis to the step functions and infer its value by fitting the case data.

$$
\begin{aligned}
\alpha_{t} & =\alpha_{t-1}+\sum_{n=1}^{m} \pi_{n} \rho_{n, t-v} \\
\tau_{t} & =\tau_{t-1}+\sum_{n=1}^{m} \pi_{n} \varphi_{n, t-v}
\end{aligned}
$$

Parameter estimation. We solve the model with a nonlinear data-fitting approach that minimizes a least squares error function as shown in Equation (12), where $F$ represents our model, $\mathbf{x}$ denotes the input data $(Q, R$ and $D)$, provided by the integration of the ordinary differential equation (ODE) system (Equations (1)-(7)) and solved with the fourth-order Runge-Kutta method. The Runge-Kutta method is easy to implement and very stable ${ }^{17} \cdot y$ denotes an observation (i.e., the reported active case number, the recovered case number, and the death case number). $\theta$ refers to all parameters $\left(\alpha, \beta, \gamma, \delta, \kappa_{0}, \kappa_{1}, \lambda_{0}\right.$ and $\left.\lambda_{1}\right)$ that are inferred by Equations (1)-(??).

$$
\min _{\theta}\|F(\theta, \mathbf{x})-y\|_{2}^{2}=\min _{\theta} \sum_{i}^{N}(F(\theta, x)-y)^{2}
$$

This function requires initial values for optimization. We set the initial values $\{0.1,2.0,0.2,0.1,0.1,3\}$ for the unknown parameters $\{\alpha, \beta, \gamma, \delta, \tau, v\}$, respectively. Some parameters are fine-tuned during the training process for each country. The optimization problem described in Equation (12) is subject to the constraints specified by the lower bound $l_{b}=[0,0,0,0,0,0]$ and the upper bound $u_{b}=[1.0,5.0,1.0,1.0,1.0,10]$. The initial values of the event impact are all set as 0.001 with the lower bound 0 and the upper bound 1 . In addition, the initial conditions of the exposed, infected and protected cases $E_{0}, I_{0}, P_{0}$ are unknown in the public dataset, which are unlikely to be zero. Hence, we set them as $0.1 Q_{0}$ since our model is not sensitive to these initial conditions. The representative measure of the optimal set of parameters is obtained with up to 2,000 iterations of optimization under the initial values and the constraint of the bounds. 
Table 4. Epidemiological attributes of the 2020 first-to-second waves in Germany.

\begin{tabular}{|l|c|c|c|c|c|}
\hline Wave & Infection rate $\beta$ & Incubation rate $\gamma$ & Quarantine rate $\delta$ & Recovery rate $\lambda(t)$ & Mortality rate $\kappa(t)$ \\
\hline $\begin{array}{l}1^{s t} \\
\text { wave }\end{array}$ & 2.0043 & 0.5760 & 0.2565 & $\begin{array}{c}(0.08)(1+ \\
\left.e^{-0.1188(t-27.22)}\right)^{-1}\end{array}$ & $0.0045\left(e^{-(0.0316(t-56.40))^{2}}\right)$ \\
\hline $\begin{array}{l}2^{\text {nd }} \\
\text { wave }\end{array}$ & 1.0558 & 0.3099 & 0.2786 & $\begin{array}{c}0.0469+ \\
e^{-0.089(t+36.06)}\end{array}$ & $\begin{array}{c}0.0022\left(e^{0.02(t-115.71)}+\right. \\
\left.e^{-0.02(t-115.71)}\right)^{-1}\end{array}$ \\
\hline
\end{tabular}

Table 5. Epidemiological attributes of the 2020 first-to-second waves in France.

\begin{tabular}{|l|c|c|c|c|c|}
\hline Wave & Infection rate $\beta$ & Incubation rate $\gamma$ & Quarantine rate $\delta$ & Recovery rate $\lambda(t)$ & Mortality rate $\kappa(t)$ \\
\hline $\begin{array}{l}1^{\text {st }} \\
\text { wave }\end{array}$ & 1.5014 & 0.4599 & 0.2749 & $\begin{array}{c}0.0027+ \\
e^{-0.0635(t+18.2)}\end{array}$ & $\begin{array}{c}0.0523\left(e^{0.09(t-29.0291)}+\right. \\
\left.e^{-0.09(t-29.03)}\right)^{-1}\end{array}$ \\
\hline $\begin{array}{l}2^{\text {nd }} \\
\text { wave }\end{array}$ & 0.6330 & 0.1507 & 0.2801 & $\begin{array}{c}0.0018(1+ \\
\left.e^{-0.0058(t-92.18)}\right)^{-1}\end{array}$ & $0.0003\left(e^{-(0.015(t-85.24))^{2}}\right)$ \\
\hline
\end{tabular}

\section{Results}

We used COVID-19 data from three European countries with typical first and second waves and their corresponding verifiable cases and interventions as the case study: Germany, France and Italy. According to the COVID-19 curves in these three countries, the first wave is specified as 1 March and 31 May 2020 and the second wave as 1 September to 1 December 2020. Both of the two wave periods are 92 days for the alignment and comparison between two waves vertically and between countries horizontally. To make the comparison more specific, we divide the parameters inferred by our model into two groups: basic epidemiological attributes, and event-sensitive variables. The epidemiological attributes are the infection rate $\beta$, the incubation rate $\gamma$, the quarantine rate $\delta$, the recovery rate $\lambda(t)$, and the mortality rate $\kappa(t)$. The event-sensitive variables are the inferred protection rate $\alpha(t)$, the deconfinement rate $\tau(t)$, the impact $\pi$ of an intervention event during its wave period, and the time delay parameter $v$ for the event to take effect. Below, we report the results and findings for the four questions above. We compare the first and second waves in terms of these epidemiological and event attributes, the COVID-19 trend forecasting in and after the second waves under various intervention strategies, the trend of infections under more infectious virus mutants, and the predictive ability of our method.

How do epidemiological attributes change over the first to second wave in different countries? The epidemiological attributes of the two waves in Germany, France and Italy in 2020 are presented in Tables 4-6, respectively. First, the infection rates of the first waves are much higher than those of the second waves, showing a higher infection probability of the susceptible individuals in the first waves, which may have lifted people's self-protection awareness in the second waves and resulted in lower infection rates. Second, the reciprocal of the incubation rate $\gamma$, namely $\gamma^{-1}$, reflects the average incubation period. The shorter incubation rates in the three countries indicate longer incubation periods in their second waves. For example, France had an average incubation period of 2.2 days in the first wave compared to 6.6 days in the second wave, inferred from their case data. The longer incubation periods may also involve more cases infected in the second waves. Third, the quarantine rates $\delta$ increased from the first waves to the second waves in both Germany and France. The exception in Italy, however, cannot be simply explained as that was caused by fewer people quarantined in the second waves. It is possible that, due to the stretched healthcare resources, many more identified infections in the second wave may have caused a relatively lower quarantined ratio.

By assuming that both the recovery rate $\lambda$ and the mortality rate $\kappa$ are time-varying, Figures (3)-(5) show the time-dependent movements of the recovery and mortality rates inferred from the case data in the three countries. In reality, the measured recovery and death rates are not constant in both the first waves which were complete and the second waves which were still evolving during that time period, although the recovery rate of the second wave in France tends to be more stable. The three countries share similar mortality changes in both waves. Specifically, in the first waves, their death rates decrease to stable after

Table 6. Epidemiological attributes of the 2020 first-to-second waves in Italy.

\begin{tabular}{|l|c|c|c|c|c|}
\hline Wave & Infection rate $\beta$ & Incubation rate $\gamma$ & Quarantine rate $\delta$ & Recovery rate $\lambda(t)$ & Mortality rate $\kappa(t)$ \\
\hline $\begin{array}{l}1^{\text {st }} \\
\text { wave }\end{array}$ & 2.2493 & 0.5724 & 0.4187 & $\begin{array}{c}0.1442(1+ \\
\left.e^{-0.0217(t-126.08)}\right)^{-1}\end{array}$ & $\begin{array}{c}0.0307\left(e^{0.0473(t-9.2992)}+\right. \\
\left.e^{-0.0473(t-9.2992)}\right)^{-1}\end{array}$ \\
\hline $\begin{array}{l}2^{\text {nd }} \\
\text { wave }\end{array}$ & 0.7307 & 0.0629 & 0.1682 & $\begin{array}{c}0.0146(1+ \\
\left.e^{0.1024(t-82.96)}\right)\end{array}$ & $0.001\left(e^{-(0.011(t-114.03))^{2}}\right)$ \\
\hline
\end{tabular}


their initial increase, while they all rise gradually in the second waves. The recovery rates are more variable than the mortality rates, which stabilize at a certain level in Germany and France, in contrast to Italy. Taking Germany as an example, as shown in Figure 3(a), the death rate increases until it reaches the peak at around 0.005 and then gradually decreases to a low level in the first wave. The recovery rate follows the same increasing trend at the beginning but then stabilizes at nearly 0.08 one month later. This phenomenon is likely attributed to the greater investment in medical resources such as PPE, ICU beds and ventilators to contain COVID-19. However, Germany's second wave shows a different trend in Figure 3(b). The death rate gradually increases and the recovery rate decreases until it reaches a stable level lower than that of the first wave. There are two possible explanations: first, the greater number of cases in the second wave overwhelmed healthcare systems; second, unlike the first wave which is complete, the second wave was still underway thus the epidemiological attributes did not stabilize yet.

In conclusion, the second waves in three countries were associated with weaker interventions but stronger deconfinement activities than their first waves. As a result of the relaxed interventions, the second waves had lower infection rates, higher quarantine rates, and longer incubation periods. These result in longer, higher and broader waves of infections in the second waves, also partially attributed to the improved awareness and practices of self-protection. However, their recovery rates and mortality rates were not necessarily lower than the first waves, particularly when relaxed interventions and restrictions on public activities occurred. This may also be related to much higher and longer infections and the resultant overwhelmed healthcare services.
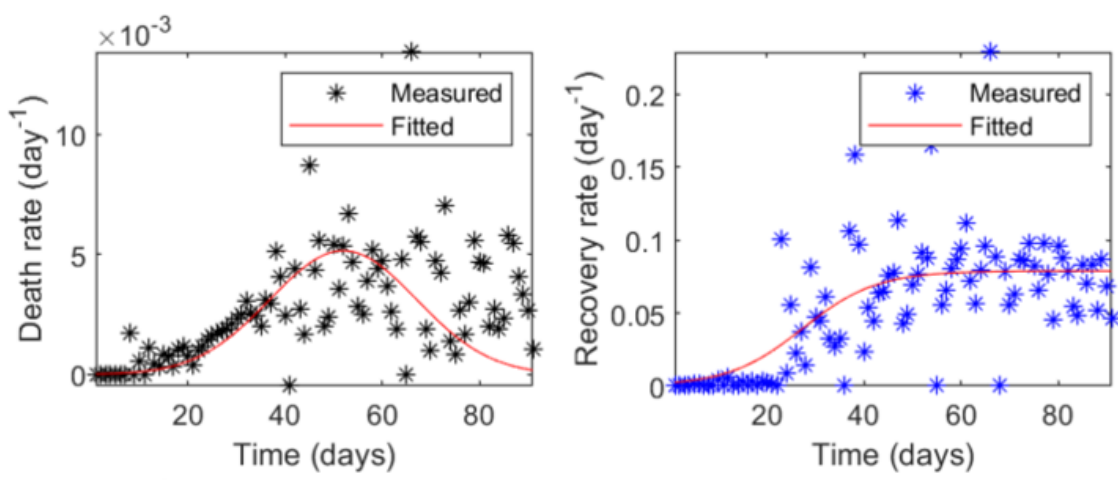

(a) The mortality and recovery rates of the first wave.
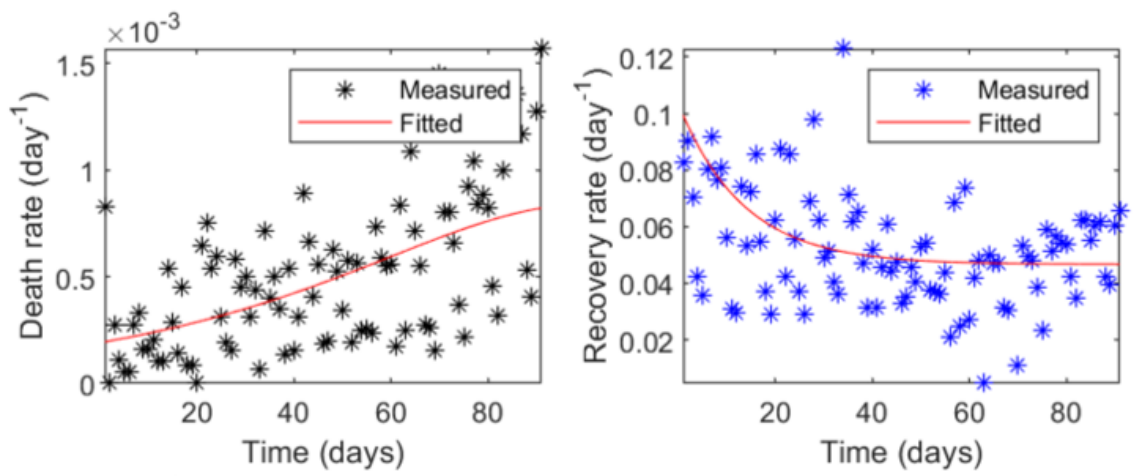

(b) The mortality and recovery rates of the second wave.

Figure 3. The time-varying recovery and mortality rates of two waves in Germany.

How does different severity, number and timing of interventions individually and cumulatively affect the trends of the two waves? To answer this question, we infer the impact of control and relaxation events on the daily cases, and compare the individual and cumulative impacts of the sequentially implemented events in the first and second waves in Germany, France and Italy. In this analysis, we only include the events that happened during the periods of the first and second waves for the alignment between epidemiological attributes and intervention impact attributes. In addition, the cases recorded between the end of the first wave and the beginning of the second wave are stable and the released policies show less significant impact on the more stabilized epidemic curves.

With the different types (control vs. relaxation), number and implementation timing (the dates) of interventions and public activities implemented over two waves in the three countries, as shown in Tables 1-3, Tables 7-9 show the inferred event impact 

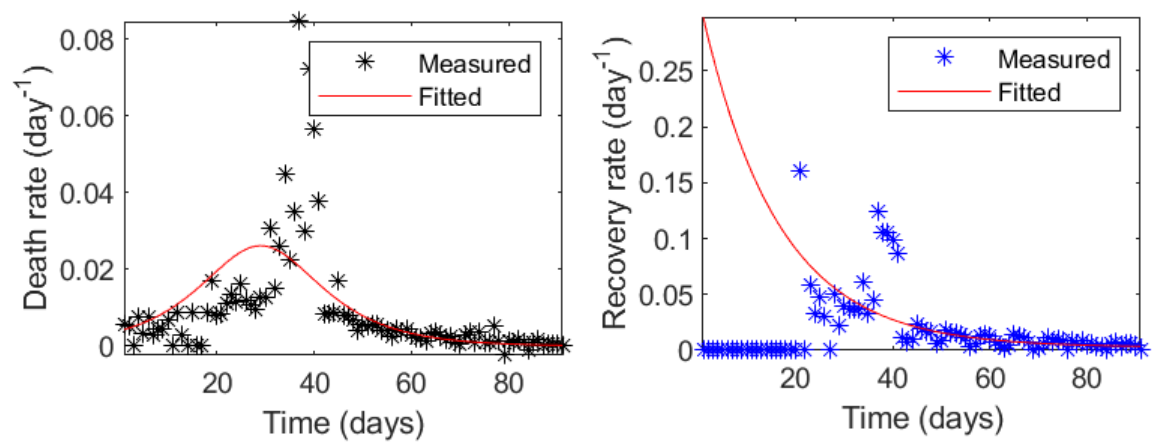

(a) The mortality and recovery rates of the first wave.
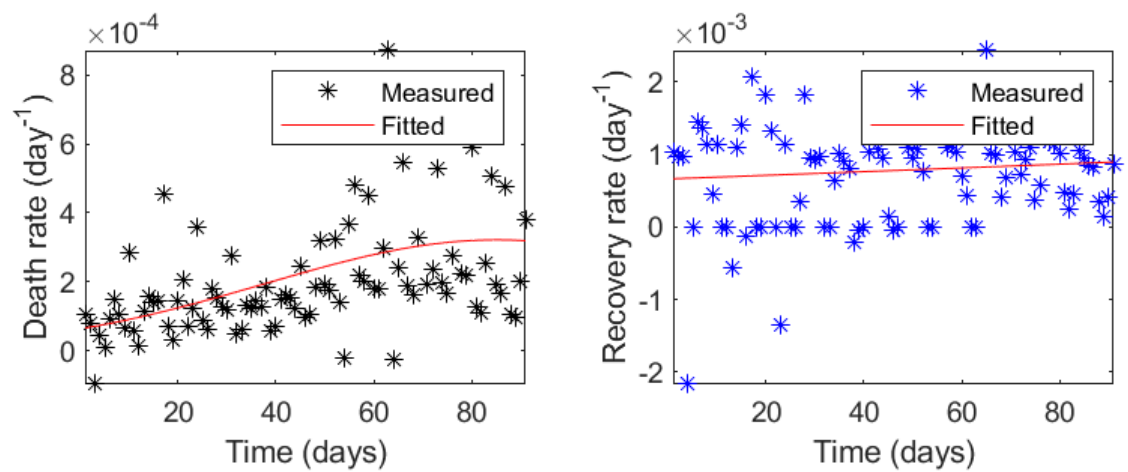

(b) The mortality and recovery rates of the second wave.

Figure 4. The time-varying recovery and mortality rates of two waves in France.
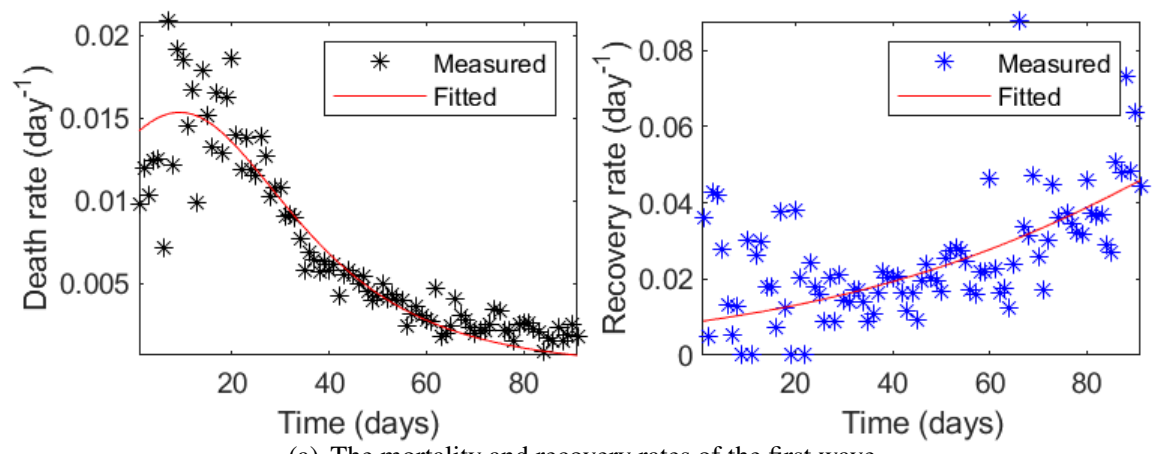

(a) The mortality and recovery rates of the first wave.
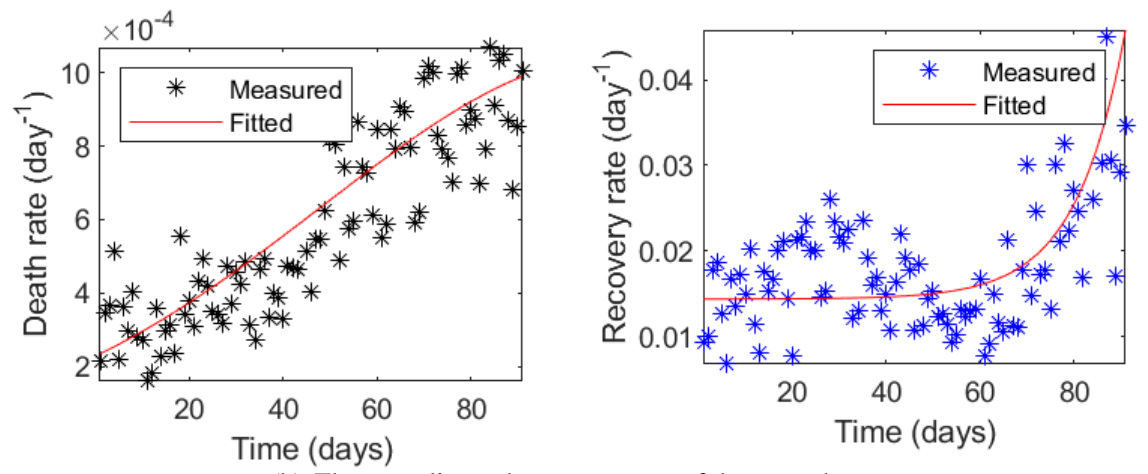

(b) The mortality and recovery rates of the second wave.

Figure 5. The time-varying recovery and mortality rates of two waves in Italy. 
attributes (the top sections) and the individual impact rate (the fitted rate value) of these events implemented in the first and second waves in each country. The parameter subscripts and superscripts in each table represent the corresponding wave number and the event number, respectively. The top section includes the event-sensitive attributes inferred by the SPEIQRD model, namely the initial protection rate $\alpha$, the initial deconfinement rate $\tau$, and the time delay $v$. The middle part shows the inferred impact $\pi$ of each event (both control and relaxation events) taking place during a wave period. The events affect the protection rates $\left(\alpha_{1}\right.$ and $\left.\alpha_{2}\right)$ and the deconfinement rates $\left(\tau_{1}\right.$ and $\left.\tau_{2}\right)$ in a cumulative way per the step functions (see Equations (10)-(11)), which cause fluctuations of the COVID-19 curves. Lastly, the overall impact of both control and relaxation events on cases is inferred at the bottom of the tables.

For an intuitive demonstration of how each event affects cases and how all events cumulatively affect cases, we align the event impact rates with the actual daily new cases in both waves in each country. First, we align each event in the first and second waves with the daily new cases on the same day in each country, as shown in Figure 6 for Germany, Figure 8 for France, and Figure 10 for Italy. In these figures, the individual impact rate of each event (the time delay factor has already been considered) is shown by the right hand vertical axis. Second, we align the cumulative impact rate of all events with the daily new cases of the first and second waves in Figure 7 for Germany, Figure 9 for France, and Figure 11 for Italy.

Below, we explain how the sequentially implemented control and relaxation events individually and cumulatively affect the daily cases in each wave, why the two waves demonstrate different dynamics and trends in each country, how control and relaxation events generate opposite effects, either suppressing or speeding up the COVID-19 curves, and how the inappropriate relaxation such as early relaxation and late intervention may have contributed to the wave differences and COVID-19 resurgence in the three countries.

In Germany, first, we discuss the wave differences in terms of the epidemiological attributes and event attributes. As shown in Table 7, a higher proportion of individuals moves directly to the protected compartment (group) in the second wave than in the first one, indicating more people had self-protection consciousness (e.g., staying at home or at other protected status such as immune or quarantined) after experiencing the first wave. This is also consistent with the reduced infection rate shown in Table 4 . The two waves have very close and low-level initial deconfinement rates $\tau_{1}$ and $\tau_{2}$, reflecting their similar average populations returning from the protected group to the susceptible compartment (e.g., losing immunity and returning to workplace, etc.) at the beginning of the two waves when there were no other relaxation events. In the second wave, there is a longer time delay for the event to take effect. Five control events and three relaxation events are included in the first wave, while three control events and one relaxation event are included in the second wave. In particular, $\pi_{2}^{1}$, as a large protest without wearing masks and maintaining social distancing, happened at the beginning of the second wave, causing deconfinement and promoting the resurgence. The interventions almost shutdown (0.1437) and stay at home (0.2056) had the most significant roles in the first and second waves, respectively.

Second, we discuss the individual impact of events occurred in the first and second waves and their relations to the two wave differences. Figure 6 shows the impact of each control or relaxation event on daily cases in the two waves. Aligning the scale of daily new cases and individual event impact in Figure 6(a) for the first wave and Figure 6(b) for the second wave shows that the events enforced in the first wave had higher containment impact than those implemented in the second wave. The first wave was quickly contained by five powerful control events implemented in the initial stage of the outbreak when the cases were low. In contrast, one significant relaxation event (a protest) was implemented in the early stage of the second outbreak, followed by two strong interventions when the cases were already high, which did not effectively contain the infection quickly, leading to a longer period, higher peak value and more cases in the second wave. The result in Figure 6 also explains the significant difference between cases and between event impacts over the two waves shown in Figure 7.

Third, we further discuss the cumulative impact of all different interventions on the wave patterns and differences between two waves. The German government had contradictory response strategies during the two waves, producing different effects on case movements as shown by the protection rate and the deconfinement rate. Figure 7(a) and Figure 7(b) further show the respective cumulative effects of all sequentially-implemented events in the two waves on the daily case movement. In the first wave, immediate and strict interventions were implemented, which were mainly adopted in the early stage of the outbreak and generated an immediate effect on flattening the curve. Relaxation measures were then made after the epidemic was substantially contained, and the deconfinement rate $\tau_{1}$ remained low in the whole first wave. In contrast, in the second wave shown in Figure 7(b), interventions lagged behind, probably because of restriction fatigue and concerns about socioeconomic recovery, the effectiveness of these late interventions was also highly discounted. A relaxation event $\pi_{2}^{1}$ (a protest) took place in the early stage, leading to the increase of the corresponding deconfinement rate $\tau_{2}$. This caused the exponential increase of daily new cases until the control events $\pi_{2}^{2}$ (stay at home), $\pi_{2}^{3}$ (partial lockdown) and $\pi_{2}^{4}$ (partial lockdown extension) took effect. In particular, the interventions $\pi_{2}^{2}$ and $\pi_{2}^{3}$ were significant in suppressing the curve rising.

In summary, the different severity, number and timing of sequentially implemented control and relaxation events in the two waves directly contribute to their distinct containment effects on infection in the two waves. The interventions implemented in the two waves led to different epidemic dynamics: the first wave was controlled quickly due to early, strong and more 
Table 7. Attributes of event impact of 2020 first-to-second waves in Germany.

\begin{tabular}{|l|c|l|c|}
\hline Attributes of $1^{\text {st }}$ wave & Fitted value & Attributes of $2^{\text {nd }}$ wave & Fitted value \\
\hline protection rate $\alpha_{1}$ & 0.0681 & protection rate $\alpha_{2}$ & 0.1644 \\
deconfinement rate $\tau_{1}$ & 0.0519 & deconfinement rate $\tau_{2}$ & 0.0501 \\
time delay $v_{1}$ & 3 & time delay $v_{2}$ & 7 \\
\hline C: cancel public events $\pi_{1}^{1}$ & 0.0320 & R: protest with mask-wearing on 20 Sep. $\pi_{2}^{1}$ & 0.0964 \\
C: school closure $\pi_{1}^{2}$ & 0.1238 & C: stay at home $\pi_{2}^{2}$ & 0.2056 \\
C: almost shutdown $\pi_{1}^{3}$ & 0.1437 & C: partial lockdown $\pi_{2}^{3}$ & 0.1464 \\
C: gathering forbidden $\pi_{1}^{4}$ & 0.1113 & C: partial lockdown extension $\pi_{2}^{4}$ & 0.0010 \\
C: mask wearing $\pi_{1}^{5}$ & 0.0624 & - & - \\
R: business reopening $\pi_{1}^{6}$ & 0.0000 & - & - \\
R: expand reopening under & 0.0140 & - & - \\
strict social distancing $\pi_{1}^{7}$ & & & \\
R: ease border control $\pi_{1}^{8}$ & 0.0259 & - & - \\
\hline overall impact of control & 0.4732 & overall impact of control & 0.3530 \\
overall impact of relaxation & 0.0399 & overall impact of relaxation & 0.0964 \\
\hline
\end{tabular}

interventions, while the second wave lasted longer and caused a higher peak number and more cases as a result of late, weak and less interventions.

In addition, though the epidemiological attributes are subject to other driving factors beyond interventions, we also obtain the following general epidemiological features of the second waves in the three countries, evidenced by the results in Tables 4-6: (1) a lower infection rate (30\% to $50 \%$ of the first waves); (2) a longer incubation period (double to ten times of the first waves); (3) a higher quarantine rate in general; (4) a higher recovery rate; and (5) a lower mortality rate. For a specific resurgence in a country or region, these findings may vary due to their particular circumstances and driving factors.

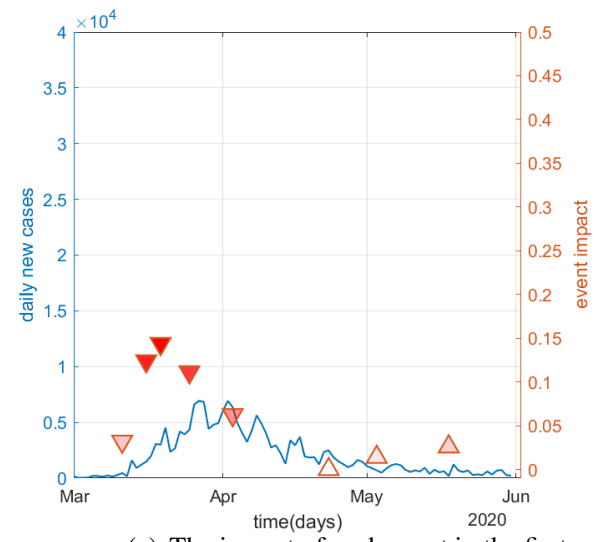

(a) The impact of each event in the first wave.

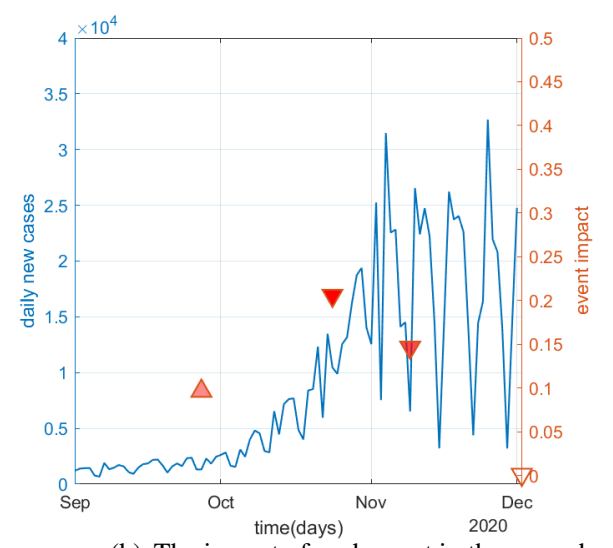

(b) The impact of each event in the second wave.

Figure 6. The individual impact of events on daily new cases during the two waves in Germany. The blue axis represents the daily new cases, and the red axis represents the event impact. The blue curve represents the change of daily new case numbers, the red regular triangles represent relaxation events, and the red inverted triangles represent control events. The deeper the color of the red triangles, the greater the impact of the events on the daily cases.

In France, first, Table 8 shows the differences between two waves in terms of their epidemiological attributes and intervention impact. The initial protection rate, the deconfinement rate and the time delay before events taking effect in the second wave were larger than that in the first wave. The increased initial deconfinement rate of the second wave may be due to the early relaxation of social restrictions. As seen in the middle section of the table, five major control events and one relaxation event were implemented in the first wave, in contrast to four major control events and one relaxing event in the second wave. As a result, after a series of interventions enforced at the beginning of the first epidemic, the infection was under control and cases declined. Then the French government reopened businesses $\left(\pi_{1}^{6}\right)$ from 12 May. This significant early relaxation may have led to the increase of the initial deconfinement rate in the second wave.

Second, we analyze the individual influences of control and relaxation interventions implemented in the two waves on the 


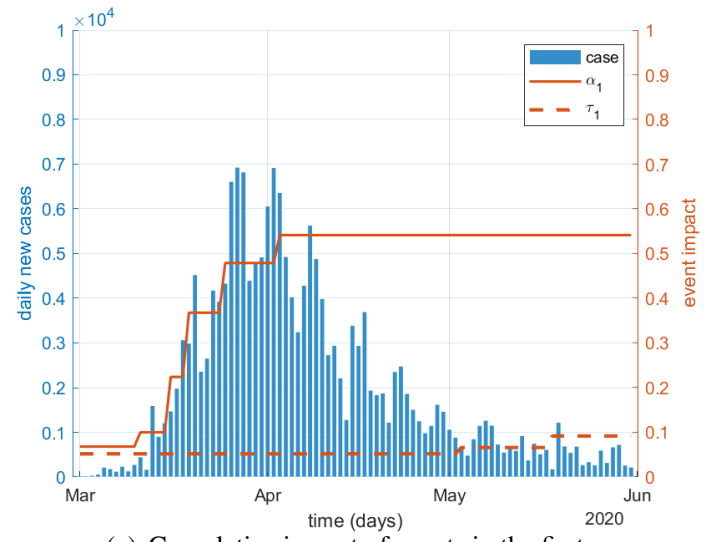

(a) Cumulative impact of events in the first wave.

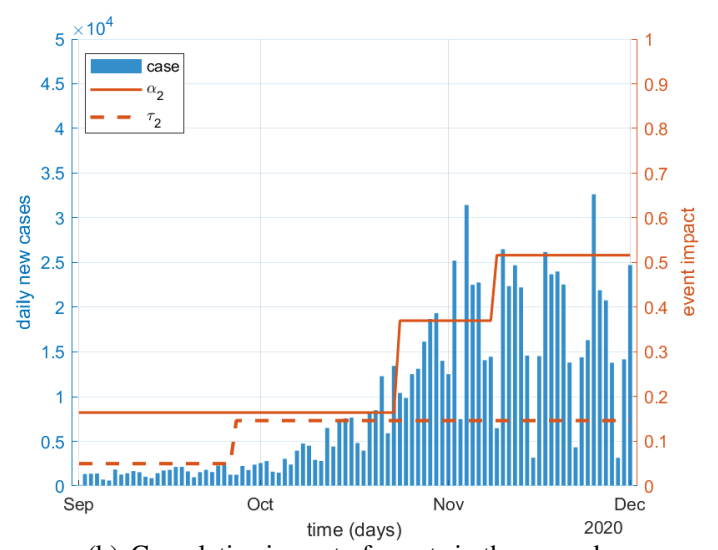

(b) Cumulative impact of events in the second wave.

Figure 7. The cumulative impact of all sequential events on daily new cases during the two waves in Germany. The blue axis represents the daily new cases, and the red axis represents the cumulative impact of sequentially-enforced events. The blue bars represent the daily new cases, the red solid line represents the cumulative impact of control events (i.e., the change of protection rate $\alpha$ ), and the red dash line represents relaxation events (i.e., the change of deconfinement rate $\tau$ ). The sequentially-implemented control and relaxation events lead to the increase of $\alpha$ and $\tau$, respectively.

different wave behaviors and results. The individual effects of control and relaxation events in the two waves in France are shown in Figure 8. In the first wave, as shown in Figure 8(a), five strong control interventions (including school closure $\pi_{1}^{4}$ and lockdown $\pi_{1}^{5}$ ) implemented in the very initial stage of the outbreak when the cases were very low generated immediate strong impact on containing cases and curves at the very beginning of the epidemic. Due to the small infectious population in the early stage, the many early and strong interventions quickly contained the first wave. However, in the second wave, as shown in Figure 8(b), the two initial interventions (mask wearing $\pi_{2}^{1}$ and gathering restriction $\pi_{2}^{2}$ ) implemented when the cases were already high (much higher than the first wave) were too mild to contain the increase of infections. The cases continuously increased and the outbreak lasted much longer with a much higher peak number of cases. This direction did not change until two strict interventions curfews $\pi_{2}^{3}$ and lockdown $\pi_{2}^{4}$ were enforced, which substantially compressed the growth to decline.

Third, we discuss the cumulative impact of all interventions on protection and deconfinement in the two waves. Figure 9 shows the protection rates and the deconfinement rates in both waves. In the first wave, the step-wise early and strong interventions were implemented in the initial stage of the outbreak when the cases were very low. They resulted in a very low deconfinement rate but a much higher protection rate and effectively and quickly contained the first wave. In contrast, the late implementation of fewer strong interventions when the cases were approaching their peak values contributed to a much lower protection rate but a much higher deconfinement rate than the first wave, where deconfinement showed stronger impact than protection. Two initial interventions mask-wearing $\pi_{2}^{1}$ and gathering restrictions $\pi_{2}^{2}$ were too soft to influence the case movement, resulted in the higher deconfinement rate than the first wave.

In summary, similar to the scenario in Germany, the different severity, number and timing of control and relaxation interventions in the two waves in France directly contributed to their different containment effects on the two waves, resulting in different case numbers, peak cases and trends in the two waves. Although the two waves were eventually under control, a longer spread period with more infections and higher peak cases was seen in the second wave in comparison to the first. In addition, the overall impact of control interventions in the second wave was much larger than that in the first wave since they took effect only until the curve reached its peak. This explains why a broader and larger population were infected in the longer second wave than the first wave.

In Italy, first, Table 9 illustrates the different epidemiological attributes and intervention impact in two waves. The initial protection rate decreased from 0.0866 in the first wave to 0.0374 in the second wave, while the initial deconfinement rate increased by nearly twice. This is different from Germany and France, which also explains that the first wave was better managed than the second wave in Italy. These inferred event parameters can be further explained by their corresponding interventions. Six control events and three relaxation events were implemented in the first wave, with the wave quickly controlled under these comprehensive strict and decisive interventions; in contrast, the five events implemented in the second wave were only for containment after a big surge of infections, resulting in a softer effect.

Second, Figure 10 explains the individual impact of the events implemented during the two waves on the daily cases. It also explains why the two waves have different numbers of infections and different daily new cases and trends, arising from the different severity, number and timing of control interventions. Similar to Germany and France, as shown in Figure 10(a), 
Table 8. Attributes of event impact of 2020 first-to-second waves in France.

\begin{tabular}{|l|c|l|c|}
\hline Attributes of the $1^{\text {st }}$ wave & Fitted value & Attributes of the $2^{\text {nd }}$ wave & Fitted value \\
\hline protection rate $\alpha_{1}$ & 0.0316 & protection rate $\alpha_{2}$ & 0.1472 \\
deconfinement rate $\tau_{1}$ & 0.0008 & deconfinement rate $\tau_{2}$ & 0.1712 \\
time delay $v_{1}$ & 1 & time delay $v_{2}$ & 4 \\
\hline C: gathering ban $(5000) \pi_{1}^{1}$ & 0.0049 & C: mask wearing in workplaces $\pi_{2}^{1}$ & 0.0003 \\
C: gathering ban $(1000) \pi_{1}^{2}$ & 0.0057 & C: gathering restriction $\pi_{2}^{2}$ & 0.0038 \\
C: gathering ban $(100) \pi_{1}^{3}$ & 0.0043 & C: overnight curfew $\pi_{2}^{3}$ & 0.2280 \\
C: school closure $\pi_{1}^{4}$ & 0.0033 & C: national lockdown $\pi_{2}^{4}$ & 0.4701 \\
C: lockdown $\pi_{1}^{5}$ & 0.0029 & R: partial services reopening $\pi_{2}^{5}$ & 0.0010 \\
R: business reopening $\pi_{1}^{6}$ & 0.0841 & - & - \\
\hline overall impact of control & 0.0211 & overall impact of control & 0.7022 \\
overall impact of relaxation & 0.0841 & overall impact of relaxation & 0.0010 \\
\hline
\end{tabular}

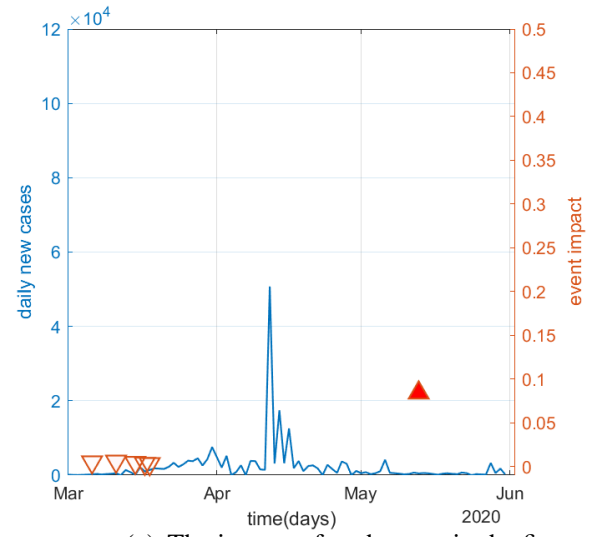

(a) The impact of each event in the first wave.

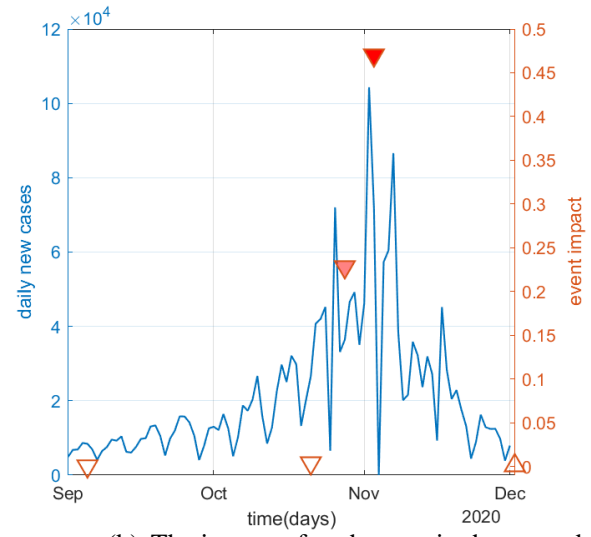

(b) The impact of each event in the second wave.

Figure 8. The individual impact of events on daily new cases during the two waves in France.

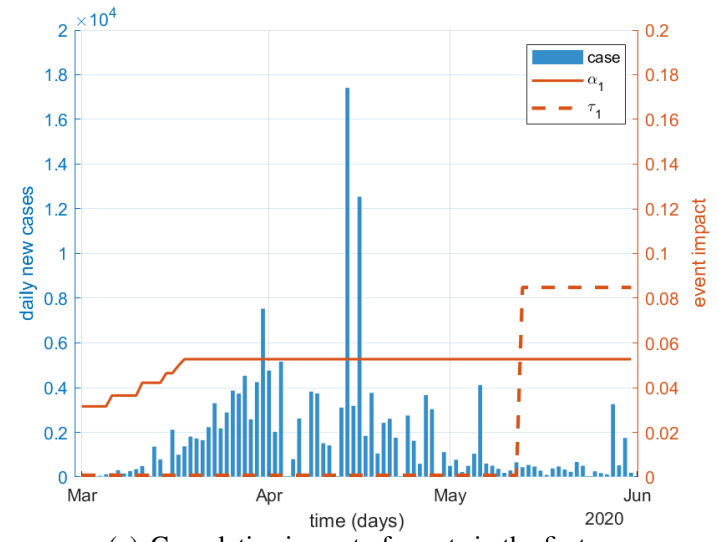

(a) Cumulative impact of events in the first wave.

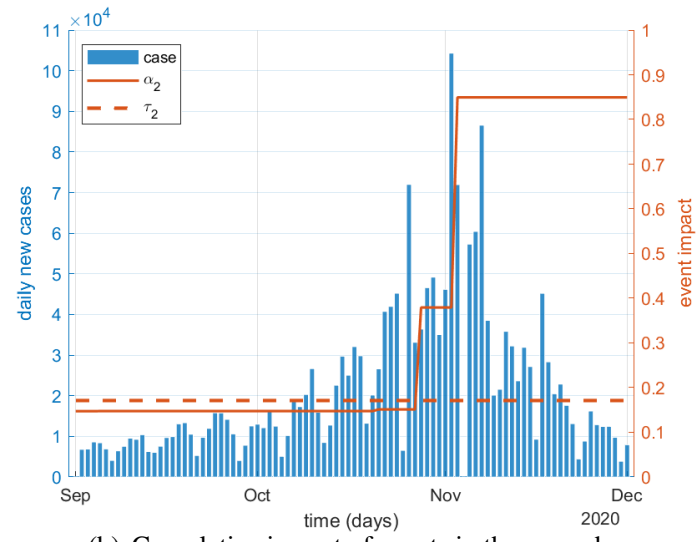

(b) Cumulative impact of events in the second wave.

Figure 9. The cumulative impact of all sequential events on daily new cases during the two waves in France. 
Table 9. Attributes of event impact of 2020 first-to-second waves in Italy.

\begin{tabular}{|c|c|c|c|}
\hline Attributes of $1^{\text {st }}$ wave & Fitted value & Attributes of $2^{\text {nd }}$ wave & Fitted value \\
\hline protection rate $\alpha_{1}$ & 0.0866 & protection rate $\alpha_{2}$ & 0.0374 \\
\hline deconfinement rate $\tau_{1}$ & 0.0769 & deconfinement rate $\tau_{2}$ & 0.1407 \\
\hline time delay $v_{1}$ & 3 & time delay $v_{2}$ & 3 \\
\hline C: local lockdown $\pi_{1}^{1}$ & 0.0307 & C: outdoor mask-wearing $\pi_{2}^{1}$ & 0.0001 \\
\hline C: school closure $\pi_{1}^{2}$ & 0.1465 & C: gathering forbidden $\pi_{2}^{2}$ & 0.0000 \\
\hline C: full lockdown $\pi_{1}^{3}$ & 0.0113 & C: school closure $\pi_{2}^{3}$ & 0.0000 \\
\hline C: lockdown tightening $\pi_{1}^{4}$ & 0.0037 & C: regional lockdown $\pi_{2}^{4}$ & 0.0290 \\
\hline C: movement restriction $\pi_{1}^{5}$ & 0.0005 & C: business shutdown $\pi_{2}^{5}$ & 0.6129 \\
\hline C: higher fines for restriction violation $\pi_{1}^{6}$ & 0.0981 & - & - \\
\hline $\mathrm{R}$ : some business reopening $\pi_{1}^{7}$ & 0.0001 & - & - \\
\hline $\begin{array}{l}\text { R: lift movement restriction and reopen indus- } \\
\text { try } \pi_{1}^{8}\end{array}$ & 0.0001 & - & - \\
\hline $\mathrm{R}:$ partial lockdown lifting $\pi_{1}^{9}$ & 0.0001 & - & - \\
\hline overall impact of control & 0.2908 & overall impact of control & 0.6420 \\
\hline overall impact of relaxation & 0.0003 & overall impact of relaxation & 0 \\
\hline
\end{tabular}

strict interventions ( $\pi_{1}^{1}$ and $\pi_{1}^{2}$ ) were adopted before the daily new cases reached 1,000. These early and strong interventions implemented in the first wave before the cases rose to a significant level effectively contained the outbreak. In contrast, as shown in Figure 10(b), effective interventions (business shutdown $\pi_{2}^{5}$ ) were not taken until the daily new cases exceeded 20,000 in the second wave. The late implementation of four interventions (including school closure $\pi_{2}^{3}$ and regional lockdown $\pi_{2}^{4}$ ) after the cases grew to a substantial level did not take effect. The cases still grew exponentially, which was only compressed after business shutdown $\pi_{2}^{5}$ was implemented at a very high case level.

Another interesting question is when to safely reopen businesses. This first wave in Italy shows some hints. From 10 April 2020, the Italian government began to relax the restrictions (cf. Table 3 and Figure 10(a)). Although the daily new cases were still considerable (more than 4,000 on average as shown in Figure 10(a)), the relaxation from 10 April to the end of May in the first wave did not lead to the infection resurgence, showing the possibility of a trade-off between epidemic containment and socioeconomic recovery at the same time after the epidemic has been substantially contained (i.e., in the decline tail stage of an outbreak). Carefully relaxing some restrictions, such as reopening business and relaxing movement restrictions, may not make the infection seriously worse and substantially change the case movement direction as long as (1) such relaxation activities are implemented after an outbreak has been fundamentally controlled and in its decline stage measures; and (2) routine control measures such as self-protection and hygiene (e.g., wearing face masks, hand sanitization, and no hand shaking) and social distancing are still enforced as 'new normal'. However, the epidemic may last for a much long period with a long tail, as shown by the case curve in Figure 10(a)).

Third, the cumulative impact of sequential interventions implemented in the two waves on daily new cases further explains the wave differences. Figure 11 shows the protection rates and the deconfinement rates over the two waves. It shows, the first wave was quickly contained since strong interventions but weak deconfinement activities were undertaken at the beginning of the outbreak. As shown in Figure 11(a), the protection rate $\alpha_{1}$ is much higher than the deconfinement rate $\tau_{1}$. In contrast, in the intial stage of the second wave, the deconfinement rate $\tau_{2}$ is higher than the protection rate $\alpha_{2}$, indicating much relaxed control on the epidemic and leading to an exponential growth of daily new cases. Accordingly, only strict interventions such as full lockdown $\left(\pi_{2}^{5}\right)$ could contain the epidemic, while it would take a longer period and cause more infections since it was implemented too late.

In summary, in Italy, the different behaviors and trends of the two waves were highly associated with their corresponding different severity, number and timing of interventions. Early, strong and more interventions at the initial stage of the outbreak effectively contained the first wave, while late, soft and less interventions resulted a longer and higher resurgence. This case study also shows that balancing epidemic containment and socioeconomic recovery is possible when the epidemic has been substantially contained, which however resulted in a longer wave with a long tailed distribution of infections. In addition, the results also show that full lockdown would be mandatory especially when there was an exponential growth of infections and the cases were at a high level.

Below, we further summarize the findings and insights of COVID-19 resurgence, including specific wave differences identified in Germany, France and Italy; the influence of control or relaxation interventions on wave differences and resurgence; effective containment strategies under different epidemic circumstances and timing; and conditions for business reopening. We 


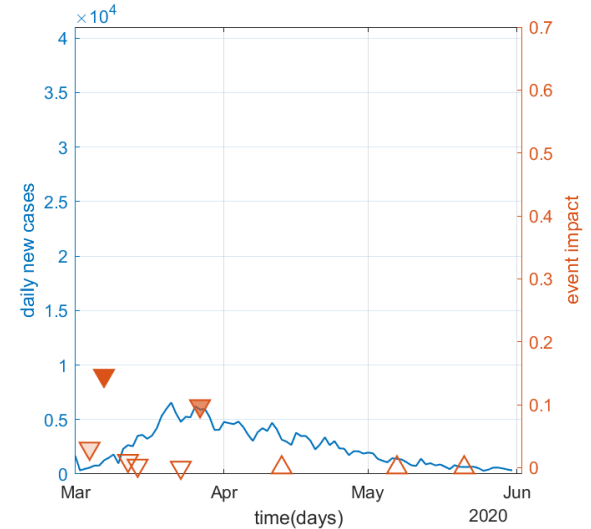

(a) The impact of each event in the first wave.

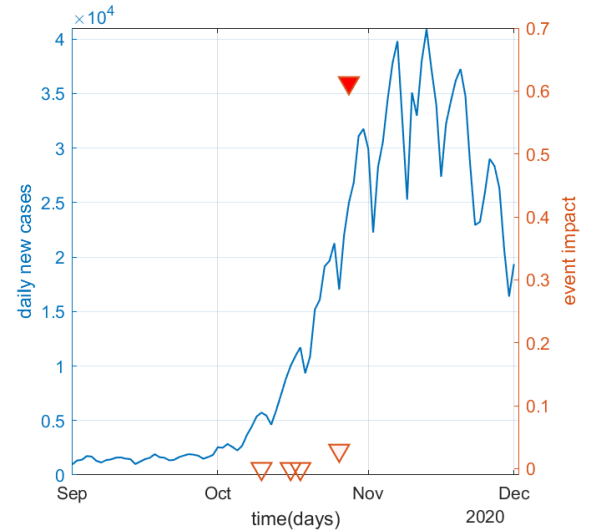

(b) The impact of each event in the second wave.

Figure 10. The individual impact of events on daily new cases during the two waves in Italy.

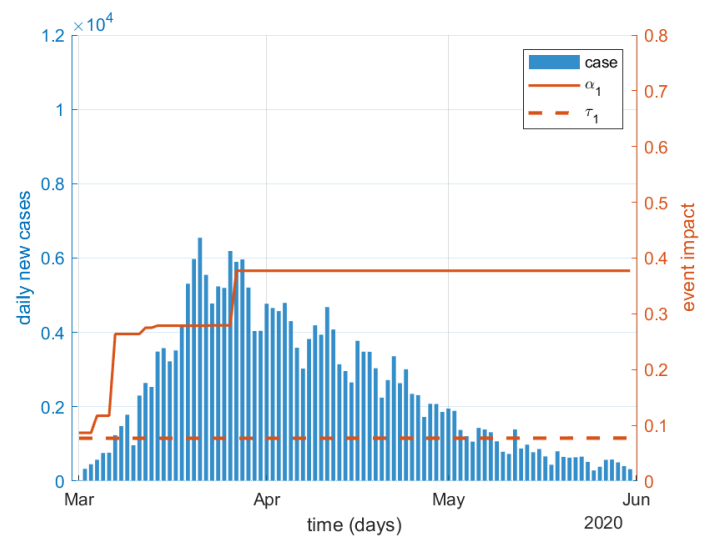

(a) Cumulative impact of events in the first wave.

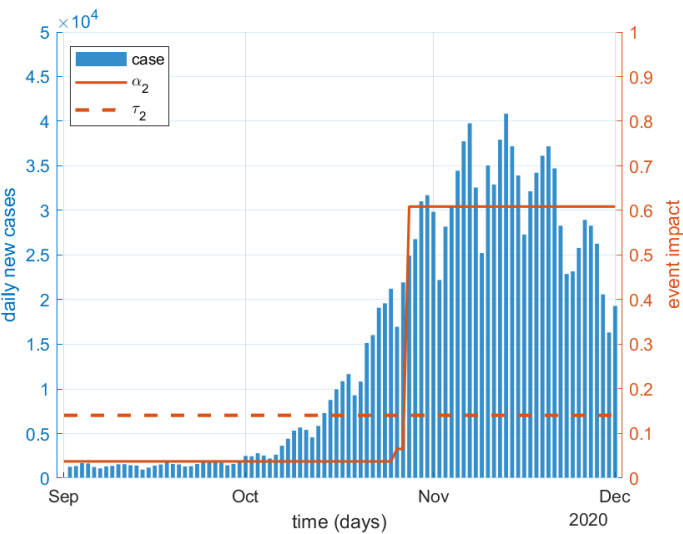

(b) Cumulative impact of events in the second wave.

Figure 11. The cumulative impact of all sequential events on daily new cases during the two waves in Italy. 
address these by combining the vertical between-wave analyses and horizontal between-country analyses and comparing the first-to-second wave evolution in the three European countries.

First, we summarize the specific wave differences both within and between countries identified in Germany, France and Italy, and also explain how different intervention strategies affect the two waves in these three countries which present both similar but also different features. (1) Better contained first waves with different wave patterns: Comparing Figure 6, Figure 8 and Figure 10, in the early stage, all three countries applied many control interventions including lockdowns which quickly contained the infection spread. However, they had different trends of daily cases, resulting from the different severity, number and timing of the interventions. Germany and Italy had a similar long-tailed movement, as a result of their late implementation of many interventions after the outbreak became more severe and the early relaxation of some interventions. In contrast, France implemented and retained several less powerful interventions at the very beginning of the first wave, resulting in a balanced single-peak distribution of cases. (2) Longer and higher second waves: The second waves demonstrated more differences than the first waves. Germany and Italy share a more similar distribution with a longer period but a lower number of infections than France, resulting from different interventions. The Germany's speedy growth of daily cases was due to their late implementation of two mild interventions, while the four very soft interventions implemented in Italy did not contain the infection until when a strong control measure business shutdown was enforced. In comparison, France had a normal distribution with contained spread within the same period as Germany and Italy by implementing two increasingly stronger control interventions overnight curfew and national lockdown, after two very soft interventions mask wearing and gathering restriction did not control the outbreak. In comparison to Germany who applied mild interventions (partial lockdown), France and Italy enforced interventions with one to multiple orders of magnitude stronger impact (such as national lockdown) to effectively control the outbreak. All the second waves show higher peak cases than the first waves across the three countries. This may be mainly caused by people's restriction fatigue and the implementation of inappropriate relaxation interventions in the second waves. In addition, the increased test rates in the second waves also contribute to the higher reported cases. The weak mitigation (and the virus mutants) may also be associated with the more frequent resurgence of cases in the second wave, making it harder to control. (3) Different severity of interventions on first-to-second wave differences: The lagged enforcing of strongly effective interventions had a prominent impact on the second waves. In the first waves of the three countries, their first control measure was taken when their daily new cases were under 1,000 ( $\leq 100$ in Germany, $\leq 200$ in France, and $\leq 600$ in Italy). In contrast, in the second waves, strong interventions were not adopted until the daily new cases became much higher (at around 10,000 in Germany, 5,000 in France and 4,000 in Italy). However, the impact of similar events differed under the distinct situations of the two waves. For example, lockdown was implemented in both the first and second COVID-19 waves in France. However, the influence on the epidemic was quite different. In the first wave, the inferred lockdown impact rate is 0.0029 , which acted more like an enhanced control intervention. In contrast, in the second wave, the impact rate reached 0.4701 , which played a decisive role in containing the epidemic. Another example is the almost shutdown intervention with an impact rate of 0.2256 in the first wave versus the similar partial lockdown with rate of 0.1464 in the second wave in Germany. However, the measure local lockdown in the first wave and regional lockdown in the second wave in Italy demonstrated similar impact of 0.0307 and 0.0290 , respectively. As partial to full lockdown is often implemented as a preferred (or even first choice) effective intervention in both waves, the effect on containment is sensitive to its severity, full lockdown in the initial stage of an outbreak may maximize the effect of early, short and low-case containment. The previous measures in the second wave in all three countries barely affected the epidemic since they are not every effective in terms of severity. This shows that it is critical for policymakers to choose the mostly effective interventions at the right time and duration for the expected consequences. (4) Different timing of interventions on first-to-second wave differences: Apart from choosing the suitable severity of an intervention, the timing of enforcing or easing an intervention such as when to reopen business needs to be calculated carefully. While relaxing restrictions addresses the cumulative negative public sentiment and expectation to mitigate the socioeconomic impacts, wrong timing (too early or too fast) particularly relaxing controls too soon or enforcing control too late, rapidly increase cases and extend the resurgence. Due to relaxed containment, after the second wave, the deconfinement level is higher than that at the end of the first wave in the three countries, indicating more people rejoined the susceptible group and had higher infection risk. This further shows the importance of managing the frequency and timing (and duration) of interventions.

Second, we discuss general findings on the severity and timing of interventions, the individual and cumulative impact of interventions, and the protection-deconfinement tradeoff of control and relaxation interventions. (1) Intervention severity: also called strictness or impact level, measures the contribution of each intervention to the containment of an epidemic. In general, interventions such as stay at home, school and business closure, partial to full lockdown, overnight curfew and high fines for restriction violation contribute to a high proportional impact, thus called strong interventions, while mask wearing, social distancing and gathering forbidden contribute to a low proportion, called soft interventions. (2) Intervention timing: The timing of implementing an intervention differentiates its impact. Early strong interventions likely result in the instant containment of an epidemic with a shorter, lower and narrower wave of infections. Late strong interventions may extend the infection to a longer period with more cases and high peak cases. (3) Individual impact: As shown in Tables 7-9, when a collection of 
interventions are implemented, each intervention contributes different proportions to influence the overall case movement. Figures 6, 8 and 10 show that a strong intervention may not work to the same proportional impact level when it is applied at different timing in relation to the curve trend. This explains why full lockdown implemented on the day with peak cases does not quickly flatten the curve as it is implemented in the initial stage of an outbreak. Similarly, a relaxation event like business reopening may not incur resurgence if it is implemented in the tail of the decline part of a curve. (4) Cumulative impact: As shown in Tables 7-9, all interventions implemented at different time points interact with each other and jointly contribute to containing an epidemic. While we can measure the overall impacts of control interventions and relaxation interventions, their difference may not distinguish whether an infection or resurgence would be quickly contained. (5) Protection-deconfinement tradeoff: The protection and deconfinement rates, shown in both Tables 7-9 and Figures 7, 9 and 11, better measure the overall influence of interventions on protection and deconfinement. The protection and deconfinement tradeoff serves as a cumulative indicator to evaluate the effectiveness of interventions, the balance between containment and relaxation, and what types of case curves a user wants to obtain.

Third, we summarize general findings on various scenarios of intervention effects on containment: early vs. late and fast vs. slow containment and discuss conditions for effective containment under different epidemic circumstances and intervention strategies. (1) Early containment: An epidemic would be quickly contained by implementing early and strong interventions at the every beginning of an outbreak when its cases are low, as shown in the first wave in France in Figure 8(a). (2) Late containment: An epidemic would be contained by implementing strong interventions in a late stage after the outbreak when its case numbers are high, as shown in the second waves in Germany, France and Italy in Figures 6(b), 8(b) and 10(b). (3) Fast containment: An epidemic with high case numbers would be contained quickly by implementing strong interventions such as strict full lockdowns, as shown in the second waves in France and Italy in Figure 8(b) and 10(b). (4) Slow containment: An epidemic with considerable case numbers would be contained slowly if weak interventions are implemented (probably also followed by strong interventions later) in the increase stage, or early and fast relaxation interventions are implemented in the decline stage when the cases are still high, as shown in the first waves in Germany and Italy in Figures 6(a) and 10(a). (5) Effective containment: The effectiveness of individual interventions (including strong interventions) is sensitive to its implementation timing and the outbreak severity. Generally strong interventions such as school closure, gathering forbidden and even full lockdown may be much less effective in quickly containing an already explosive outbreak compared to early interventions in the initial stage of the outbreak with low cases. For an early and fast containment of an outbreak, called early effective containment, implementing and combining strong interventions such as hotspot-based full lockdown with school closure and stay at home are much more effective than many soft interventions such as mask wearing and social distancing. To contain an already severe epidemic, full lockdown together with control measures like business and school shutdowns, banning public activities and staying at home is the only effective option. This forms a late effective containment.

Four, we summarize the general reasons for wave differences and COVID-19 resurgence and introduce the protectiondeconfinement conflict to judge whether a resurgence is possible. (1) Wave differences: As shown in Figures 6, 8 and 10, different strictness, timing and number of interventions likely contribute to different epidemic features of different waves in a country and between countries; a normally-distributed case curve is likely associated with early strong interventions without significant early and fast relaxation of these interventions; in contrast, lagging and soft interventions would result in an exponential distribution of an outbreak for a longer period of curve with higher peak cases; prematurely early and fast relaxation of interventions would extend the curve to a long tailed distribution. (2) Resurgence: In addition to other causes such as imported infections, Figures 6,8 and 10 also show that, prematurely relaxing or lifting strong interventions, early and fast reopening, and more infectious virus mutants may likely expand infection channels and cases or even change the case movement direction from a downturn to a uprising trend. (3) Protection-deconfinement conflict: As shown in Figures 7, 9 and 11, the protection-deconfinement conflict determines the non-normal curve distribution of cases. A resurgence may occur when the deconfinement rate (corresponding to highly relaxed or lifted circumstances) is substantially higher than the protection rate; higher difference more rapid growth of the cases. In contrast, when the protection rate is much higher than the deconfinement rate, the curve will be flattened.

Lastly, we discuss the conditions for achieving a tradeoff between containment and relaxation (the containment-relaxation tradeoff) and for business reopening. (1) Containment-relaxation tradeoff in the increase stage: It would be difficult (and risky) to reach a balance between effective containment and socioeconomic recovery in the increase stage of an outbreak since either late and soft intervention or early and fast relaxation may cause an exponential growth of cases, as shown in the second wave in Germany in Figure 6(b). A balance of "living with the virus" may be possible in the increase stage of an epidemic only when considerable infection cases (with sufficient healthcare resources) and moderate socioeconomic activities (with good self protection and mild interventions such as mask-wearing, social distancing and large gathering bans) are acceptable. (2) Containment-relaxation tradeoff in the decline stage: In reality, socioeconomic recovery such as business reopening should mainly be pursued in the decline stage of an outbreak case development. Similar balance and arrangements as in the above increase stage would be more feasible in the decline stage, as shown in Figure 10(a), while such early reopening would result in 
a long tailed decline trend of infections. (3) Full reopening: It would be safe to fully reopen businesses and the societies when the cases are extremely low (in comparison with the sufficient affordability of public healthcare resources and the reproduction number is less than 1) and good hygiene and self protection such as social distancing and mask wearing remain suggested, as shown in Figures 6(a) and 8(a).

The above summary of specific and general findings provide a tool for appropriately managing control and relaxation interventions, evaluating inappropriate interventions and their relaxation, estimating the impact of interventions on cases and the resurgence behaviors and trends. They can also guide policy-makers in making tradeoffs between epidemic containment and socioeconomic recovery, differentiating between relaxation and control interventions, and determining the timing of interventions.

How would different control and relaxation intervention strategies influence the next 30-day trends following the second waves? The above analysis highlights the important role of interventions, as shown by the protection rate $\alpha$ and deconfinement rate $\tau$ of the spread of COVID-19. Here, we further explore how the epidemic would proceed in the next 30 days following the last day of the second wave if different control-relaxation interventions are undertaken. Specifically, we simulate the case trends under either stricter control measures or more relaxed social recovery. To simulate these opposing interventions and their effects, we vary $\alpha^{\prime}$ and $\tau^{\prime}$ from their current values $\alpha$ and $\tau$ to the maximum value 1 . Since these rates are driven by events, the change of $\alpha$ and $\tau$ reflects the different response strategies by governments, individuals and societies to the epidemic. For example, if a government implements new control interventions, more susceptible individuals will be protected, resulting in the increase of the protection rate. Hence, it is conversely true that an increased $\alpha$ value indicates more control events. Similarly, an increased $\tau$ value indicates the implementation of relaxation events because more individuals in the protected and confinement groups are liberated and then return to the susceptible population. Therefore, by manipulating $\alpha$ and $\tau$, we simulate different scenarios of government interventions on containing or tolerating COVID-19.

Six different scenarios are explored for each country to forecast their effects on cases: (a) maintaining both $\alpha$ and $\tau$ at the current level; (b)-(c) increasing $\alpha$ but keeping $\tau$ unchanged; (d)-(e) keeping $\alpha$ unchanged but increasing $\tau$; and (f) increasing both $\alpha$ and $\tau$. The simulation results are shown in Figures 12-14. When increasing the protection rate as in scenarios (b) and (c), $\alpha^{\prime}$ will easily exceed its upper bound (the maximum 1) since the initial value $\alpha$ has been at a relatively high level. Hence, we set $\alpha^{\prime}=1.0$ (the maximum) if it is larger than 1. This simulates the situation that all the susceptible individuals flow into the protected compartment, indicating that the strictest control interventions are adopted.

The simulation shows that, if the current control interventions remain unchanged, namely $\alpha^{\prime}=\alpha$ and $\tau^{\prime}=\tau$ as shown in scenario (a) in Figures 12-14, the daily new cases continue to decline in the following 30 days. This means that the wave will be under control with the existing interventions which are relatively weak and allow certain socioeconomic recovery activities. Alternatively, if the protection rate increases (e.g., by 50\% as shown in Figure 12(b), 10\% in Figure 13(b), and 40\% in Figure 14(b)) or reaches its maximum 1.0 (i.e., the scenario (c) in Figures 12-14), more susceptible people will be protected and the epidemic will be better contained more quickly.

Conversely, when the protection rate remains unchanged (i.e., $\alpha^{\prime}=\alpha$ ) and we only increase the deconfinement rate by $50 \%$ (i.e., the scenario (d) in Figures 12-14) or 100\% (i.e., the scenario (e) in Figures 12-14), with more relaxation interventions, the daily new case curves show different trends in the three countries. In Germany, the curve continues to decrease in a short time period and then turns to an upward trend (see Figure 12). A larger deconfinement rate implies a faster growth of daily new cases. Undoubtedly, there would be another peak of the daily new cases in scenarios (d) and (e) in Germany. In contrast, in France, the daily new cases continue to decrease but the decrease will slow down. This is because, compared with the protection rate, the deconfinement rate at the end of the second wave is quite low $(\tau=0.1722)$, even when it doubles (i.e., $\left.\tau^{\prime}=2.0 \tau=0.3444\right)$. Consequently, the epidemic will still be contained by the control interventions and a larger protection rate. Small growth of the deconfinement rate will not affect the trend of the daily new case curve. However, in Italy, as shown in Figure 14, the curve will decrease first and then become stable when the deconfinement rate increases by $50 \%$ in scenario (d). When it increases to $\tau^{\prime}=2.0 \tau$, the daily new cases will start to increase after a short-term drop, as shown in Figure 14(e). Further, we evaluate a relatively balanced strategy by increasing both the protection rate and the deconfinement rate, as shown in scenario (f) in Figure 12-14. This aims to control the epidemic but resume social activities at the same time,such as by reviving society under strict interventions like social distancing and mask wearing. Accordingly, when we increase the two rates to their maximum values for all three countries, their daily new case curves go upwards quickly after a short-term decrease in the first few days.

We also evaluate the effect of adjusting the six different scenarios on the confirmed cases and the daily active cases following the second waves in the three countries. The confirmed cases increase in all scenarios. The change of daily active cases results from the daily new cases, the recovery rate, and the mortality rate, which show different trends in these countries.

In conclusion, it would not be safe to reopen society at a large scale at the end of the second wave (i.e., the beginning of December 2020) when there would still be considerable infected individuals. With only the limited interventions mixed with relaxation events, too early or fast relaxation of interventions would definitely return society to the pandemic outbreak.

How would more infectious virus mutants influence the second waves under hard or soft interventions? As shown in 


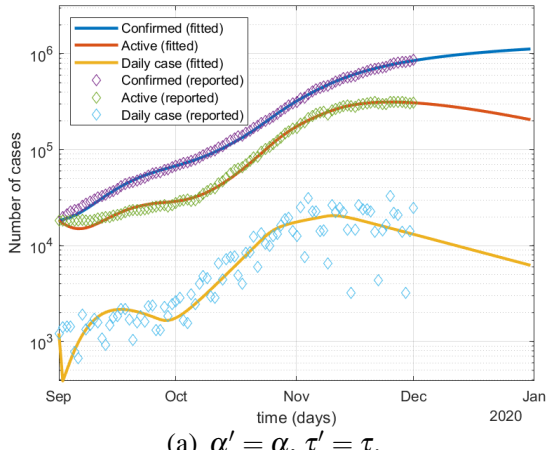

(a) $\alpha^{\prime}=\alpha, \tau^{\prime}=\tau$

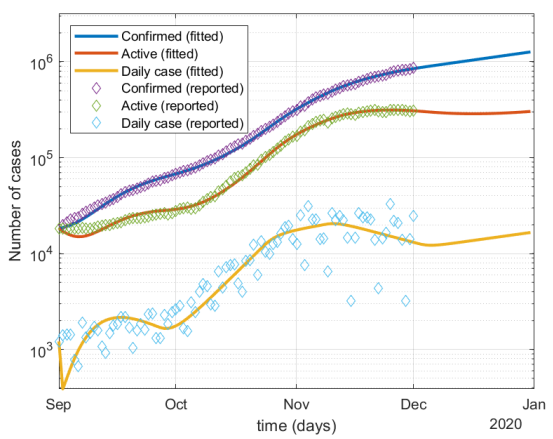

(d) $\alpha^{\prime}=\alpha, \tau^{\prime}=1.5 \tau$.

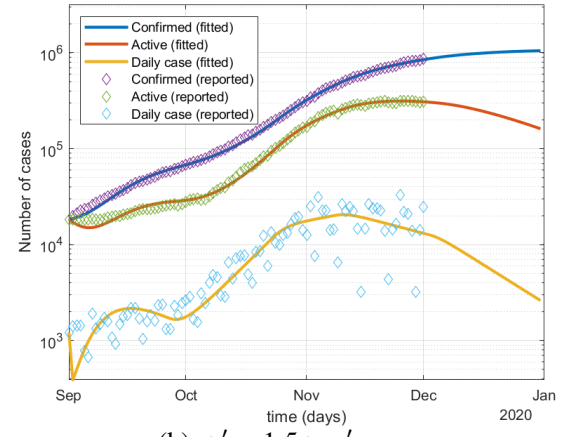

(b) $\alpha^{\prime}=1.5 \alpha, \tau^{\prime}=\tau$.

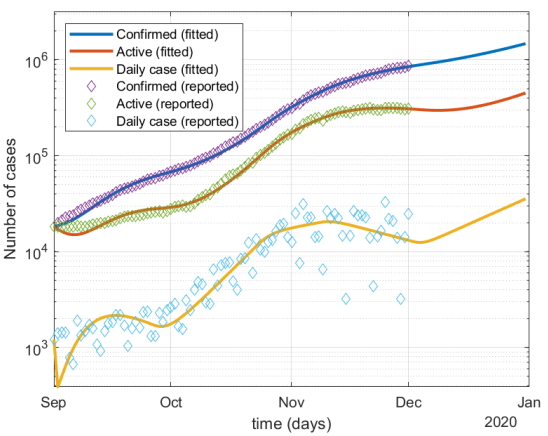

(e) $\alpha^{\prime}=\alpha, \tau^{\prime}=2.0 \tau$.

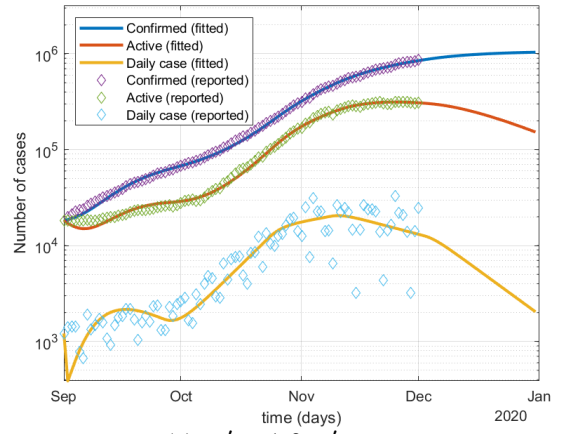

(c) $\alpha^{\prime}=1.0, \tau^{\prime}=\tau$.

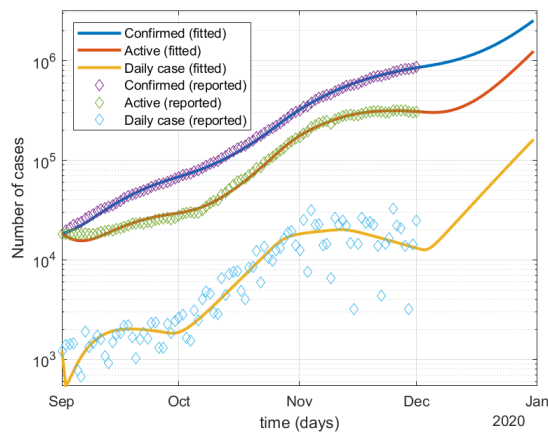

(f) $\alpha^{\prime}=1.0, \tau^{\prime}=1.0$.

Figure 12. The prediction of the next 30-day infection trend following the second COVID-19 wave in Germany by relaxing or enforcing interventions. (a)-(f) simulate six scenarios corresponding to different control and relaxation interventions with respective protection rates $\alpha$ and deconfinement rates $\tau$. The diamond markers are reported data and the solid lines are the fitted data. The confirmed cases (blue line and purple diamond), the daily active cases (red line and green diamond) and the daily new cases (orange line and blue diamond) are forecasted. 


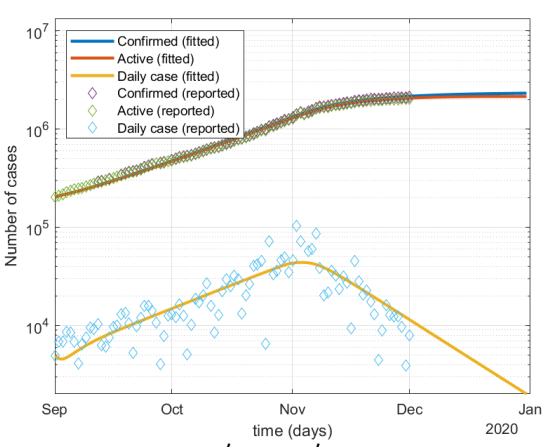

(a) $\alpha^{\prime}=\alpha, \tau^{\prime}=\tau$.

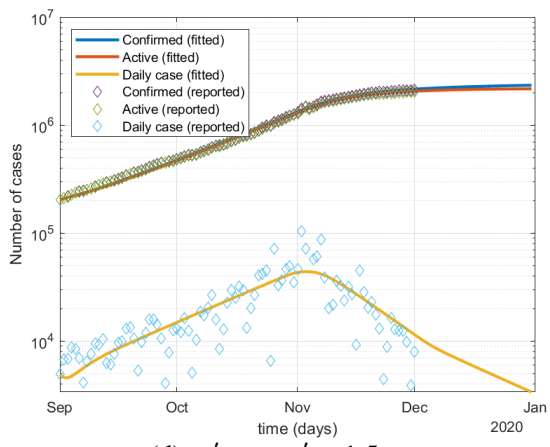

(d) $\alpha^{\prime}=\alpha, \tau^{\prime}=1.5 \tau$.

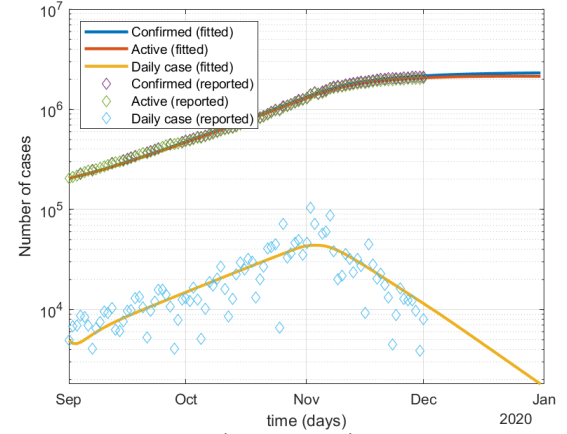

(b) $\alpha^{\prime}=1.1 \alpha, \tau^{\prime}=\tau$

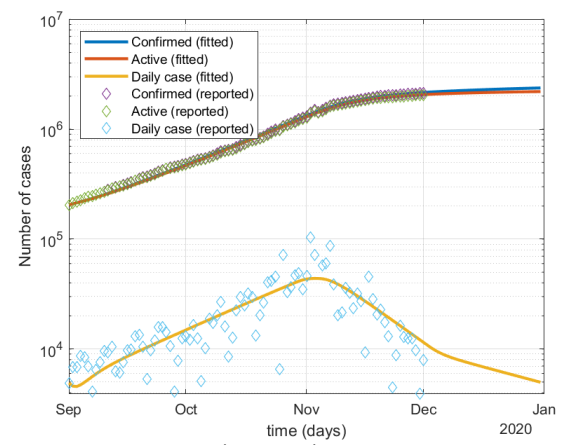

(e) $\alpha^{\prime}=\alpha, \tau^{\prime}=2.0 \tau$

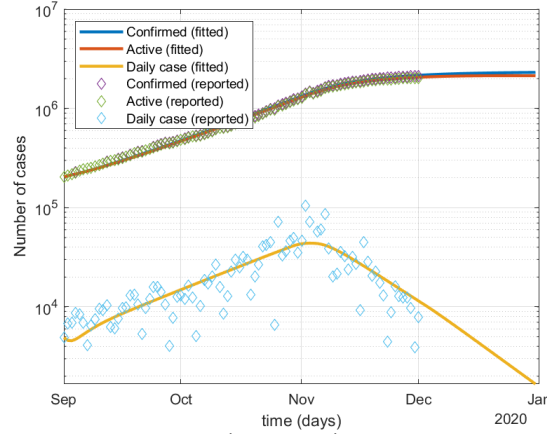

(c) $\alpha^{\prime}=1.0, \tau^{\prime}=\tau$

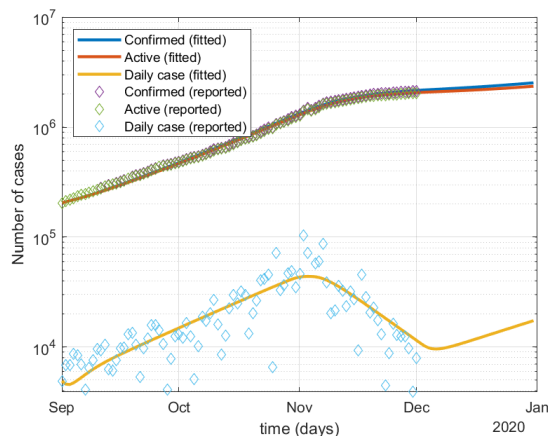

(f) $\alpha^{\prime}=1.0, \tau^{\prime}=1.0$.

Figure 13. The prediction of the next 30-day infection trend following the second COVID-19 wave in France by relaxing or enforcing interventions.

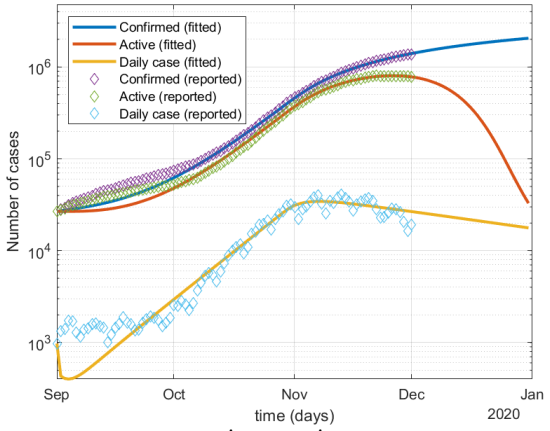

(a) $\alpha^{\prime}=\alpha, \tau^{\prime}=\tau$.

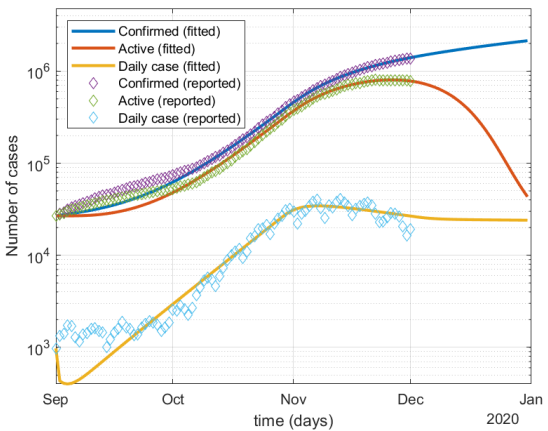

(d) $\alpha^{\prime}=\alpha, \tau^{\prime}=1.5 \tau$.

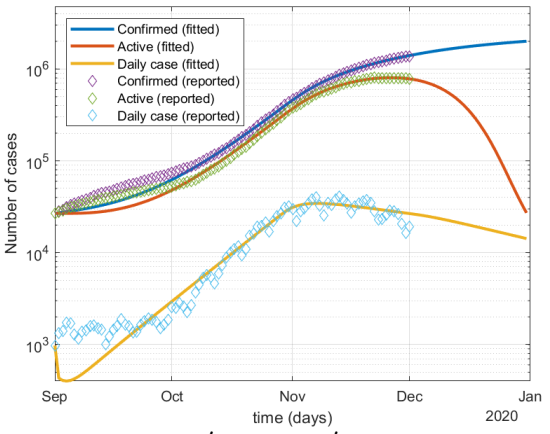

(b) $\alpha^{\prime}=1.4 \alpha, \tau^{\prime}=\tau$.

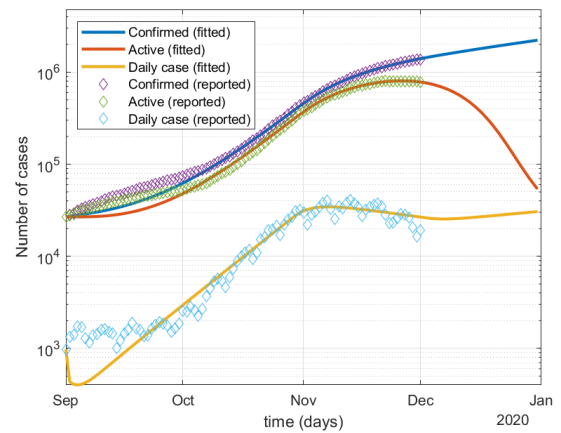

(e) $\alpha^{\prime}=\alpha, \tau^{\prime}=2.0 \tau$.

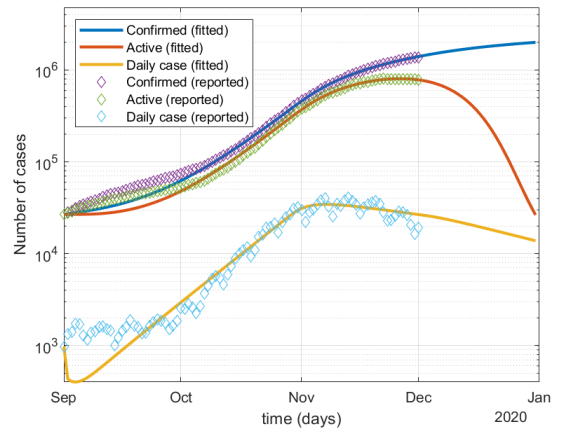

(c) $\alpha^{\prime}=1.0, \tau^{\prime}=\tau$.

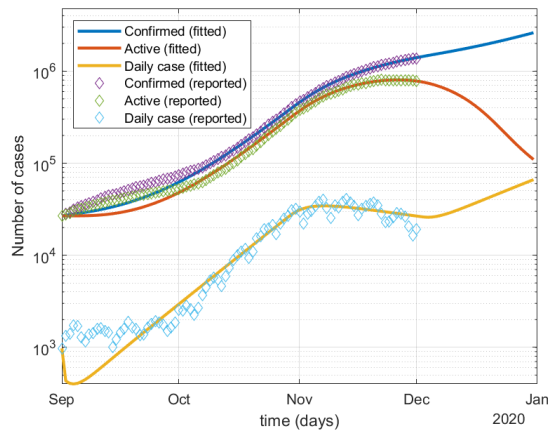

(f) $\alpha^{\prime}=1.0, \tau^{\prime}=1.0$.

Figure 14. The prediction of the next 30-day infection trend following the second COVID-19 wave in Italy by relaxing or enforcing interventions. 
practice, interventions play an important role in changing COVID-19 infection trends and spread particularly when vaccination is low and public health resources are stretched. The first waves in most European countries were successfully contained after rapidly implementing effective interventions. However, in the second waves, most governments hesitated to implement strict control measures after experiencing strict measures in the first wave, resulting in a longer duration and more cases. Here, we simulate two scenarios: whether tightening interventions would change the attributes of the second waves, and what may happen if more infectious virus mutants (such as delta or lambda) appeared in the second wave.

First, we explore the trend of the second waves if the same interventions which successfully contained the first waves were carried forward and compare the simulation results with the actual second waves in each country. This indicates how tightening versus relaxing containment would affect cases. Specifically, we simulate the daily cases by applying the same interventions which were effective in the first wave and observe to what extent the second wave would be controlled with the experience learned from the first wave. The results are shown in Figure 15 for Germany, France and Italy.

For the first scenario, when the infection rate remains unchanged (i.e., no more infectious mutants), $\beta^{\prime}=\beta$, the daily cases over the second wave would decrease dramatically if the interventions applied to the first waves were implemented, as shown in Figure 15(a) and (c) in Germany and Italy. This demonstrates the effectiveness of applying the strong interventions in the first wave to substantially control the second wave, as the relaxation in the second wave did lead to unexpected spread. In contrast, in France, the daily new case curve did not show a significant deviation from the reported until the turning point at the beginning of November, as shown in Figure 15(b). The large increase of cases leads to a great gap and difference between the two waves in France and the simulation shows that, even if the same interventions as the first wave were implemented in the second wave, the control intensity is still insufficient to quickly and effectively contain the severe second wave, probably requiring stronger and earlier interventions.

For the second scenario, we assume more infectious virus mutants appeared in the second waves by increasing the transmission rates by $20 \%, 50 \%$ and $100 \%$ (i.e., $\beta^{\prime}=1.2 \beta, \beta^{\prime}=1.5 \beta$ and $\beta^{\prime}=2.0 \beta$ ) ${ }^{7}$. We first simulate the effect on the daily cases by transferring the same interventions from the first to the second waves. Figure 15 shows the daily cases under these three mutation scenarios after directly applying the strong interventions from the first waves. Similar to the above analysis, similar trends are observed in the three countries, though more transmissible mutants like delta and lambda would substantially increase the daily cases. For example, as shown in Table 10, in France, a 50\% transmissibility increase would lift the peak cases by 7 times to 880,925 cases, while a $100 \%$ infection rate increase would result in 12 times of increase of the peak value to $1,385,640$ cases infected on the peak day. In addition, in Germany, after 2-3 weeks, the daily new cases with the three mutants would reach their peaks at 3,226, 6,692 and 20,678, i.e., increasing 0.9 times, 3.2 times and 11.3 times compared with the actual observed daily new cases contemporaneously. In Italy, the daily new cases for the $2.0 \beta$ mutant would reach 100,079 , i.e., increasing 4.2 times at the end of the period. The more contagious mutant would be eventually controlled after making strong interventions. In Germany, the daily cases with the three mutants would decrease to 1, 9, and 208 at the end of the period. In Italy, the cases would reach 4,397 for $1.2 \beta$ (decreasing by $77 \%$ ) or 16,048 for $1.5 \beta$ (decreasing by $17 \%$ )). In France, a second wave with a more infectious virus would see many more cases (e.g., increase of 3.1 times, 8.4 times and 13.3 times for the three mutants maximally), and much stronger and earlier interventions would be essential compared to Germany and Italy.

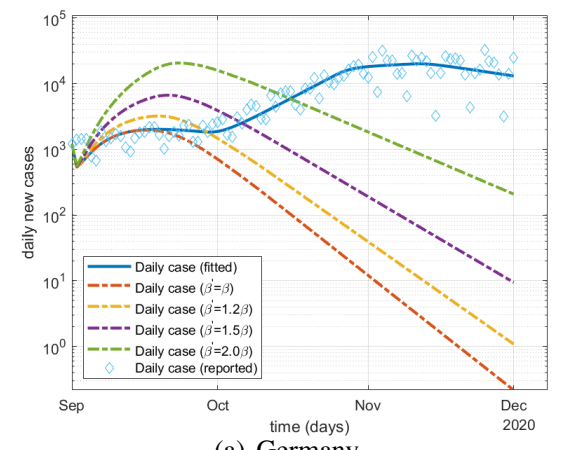

(a) Germany

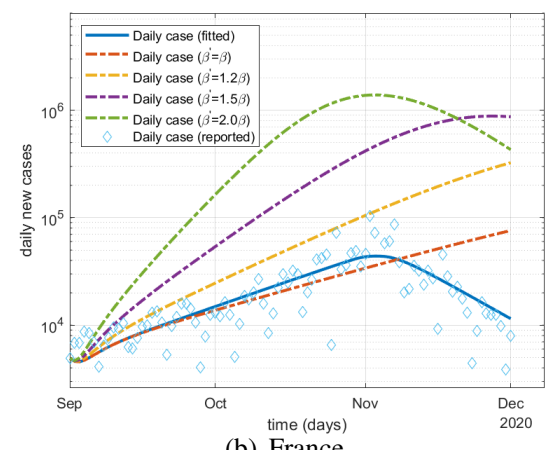

(b) France

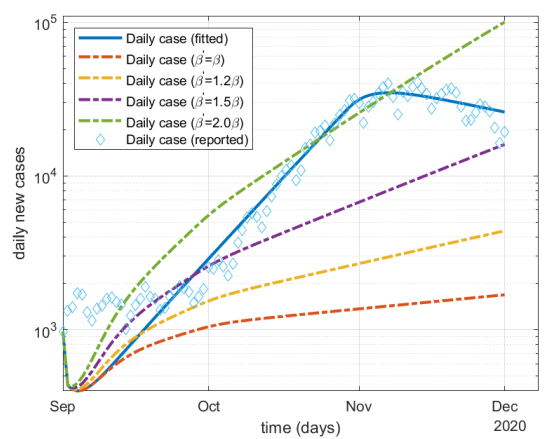

(c) Italy

Figure 15. Simulation of the second wave with strong interventions (i.e., the same control interventions as in the first wave) but with more infectious virus mutants.

Further, we simulate the trends of daily cases under more infectious virus mutants in the second wave without changing strategies, i.e., keeping the same relaxation interventions as implemented in the three countries. Figure 16 shows the simulation

\footnotetext{
${ }^{7}$ Here, we assume the remaining epidemiological attributes such as mortality rate remain unchanged
} 
Table 10. Change of peak daily cases in the second wave caused by more infectious mutants with strong interventions (i.e., the same as in the first waves) in three European countries, corresponding to Figure $15 . \Delta_{1}$ refers to the change of daily cases on the estimated peak day compared to the first day of the second wave; $\Delta_{2}$ refers to the change of daily cases on the estimated peak day compared to the actual peak value of the second wave.

\begin{tabular}{|c|c|c|c|c|c|c|}
\hline Country & First Day in $2 n d$ Wave & Observed peak value & $1.0 \beta$ & $1.2 \beta\left(\Delta_{1} ; \Delta_{2}\right)$ & $1.5 \beta\left(\Delta_{1} ; \Delta_{2}\right)$ & $2.0 \beta\left(\Delta_{1} ; \Delta_{2}\right)$ \\
\hline Germany & 1,213 & 32,687 & $1,963(\uparrow 62 \% ; \downarrow 94 \%)$ & $3,226(\uparrow 166 \% ; \downarrow 90 \%)$ & $6,691(\uparrow 452 \% ; \downarrow 79 \%)$ & $20,677(\uparrow 1605 \% ; \downarrow 37 \%)$ \\
\hline France & 4,898 & 104,314 & $75,940(\uparrow 1450 \% ; \downarrow 27 \%)$ & $324,337(\uparrow 6522 \% ; \uparrow 68 \%)$ & $880,925(\uparrow 17885 \% ; \uparrow 744 \%)$ & $1,385,640(\uparrow 28190 \% ; \uparrow 1228 \%)$ \\
\hline Italy & 975 & 34,762 & $1,687(\uparrow 73 \% ; \downarrow 95 \%)$ & $4,397(\uparrow 351 \% ; \downarrow \vee 87 \%)$ & $16,048(\uparrow 1546 \% ; \downarrow 54 \%)$ & $100,079(\uparrow 10164 \% ; \uparrow 188 \%)$ \\
\hline
\end{tabular}

Table 11. Change of peak daily cases in the second wave caused by more infectious mutants with soft interventions (i.e., the same as in the first waves) in three European countries, corresponding to Figure 16. $\Delta_{1}$ refers to the change of daily cases on the estimated peak day compared to the first day of the second wave; $\Delta_{2}$ refers to the change of daily cases on the estimated peak day compared to the actual peak value of the second wave.

\begin{tabular}{|c|c|c|c|c|c|}
\hline Country & First Day in $2 n d$ Wave & $1.0 \beta$ & $1.2 \beta\left(\Delta_{1} ; \Delta_{2}\right)$ & $1.5 \beta\left(\Delta_{1} ; \Delta_{2}\right)$ & $2.0 \beta\left(\Delta_{1} ; \Delta_{2}\right)$ \\
\hline Germany & 1,213 & 20,115 & $154,387(\uparrow 12627 \% ; \uparrow 667 \%)$ & $1,086,409(\uparrow 89464 \% ; \uparrow 5301 \%)$ & $3,022,314(\uparrow 249060 \% ; \uparrow 14925 \%)$ \\
\hline France & 4,898 & 43,896 & $142,600(\uparrow 2811 \% ; \uparrow 225 \%)$ & $560,650(\uparrow 11346 \% ; \uparrow 1177 \%)$ & $1,492,105(\uparrow 30363 \% ; \uparrow 3299 \%)$ \\
\hline Italy & 975 & 34,762 & $96,398(\uparrow 9787 \% ; \uparrow 177 \%)$ & $342,748(\uparrow 35054 \% ; \uparrow 886 \%)$ & $1,150,504(\uparrow 117900 \% ; \uparrow 3210 \%)$ \\
\hline
\end{tabular}

results when increasing the infection rate by $20 \%, 50 \%$ and $100 \%$. Under relatively soft interventions as implemented in the second wave in Germany, France and Italy, the daily new cases would increase significantly, as shown in Table 11. For example, with $100 \%$ transmissibility increase, on the peak day, 3,022,314 Germans (3.8\%), 1,492,105 French people (2.2\%), and 1,150,504 Italians (1.9\%) would be infected under the same interventions implemented for the second waves, such as stay at home, partial lockdown, mask wearing, gathering restrictions and overnight curfew. Specifically, the peak value would increase 7 times, 53 times and 149 times in Germany, resulted from 20\%, 50\% and 100\% increase of infection. France would see 2 times, 12 times and 33 times of peak case increase, respectively. In Italy, the peak value would increase 2 times, 9 times and 32 times in Italy due to the stronger transmissibility of the mutants. We also find that, when the daily new cases achieve the peak value, the higher the transmissibility, the faster the infection falls. This may be because, with higher transmissibility and looser containment, there would be substantially more cases and many fewer susceptible individuals left, leading to much stronger herd immunity of the population.

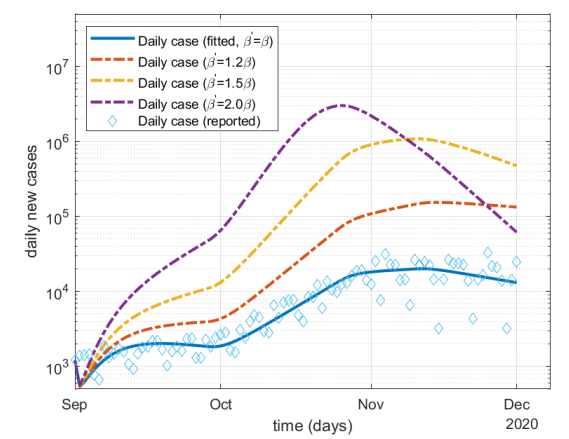

(a) Germany

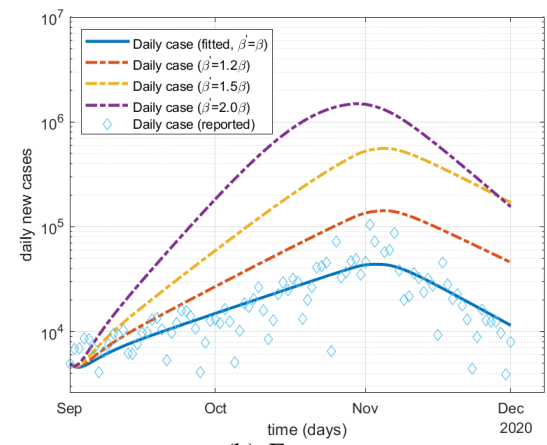

(b) France

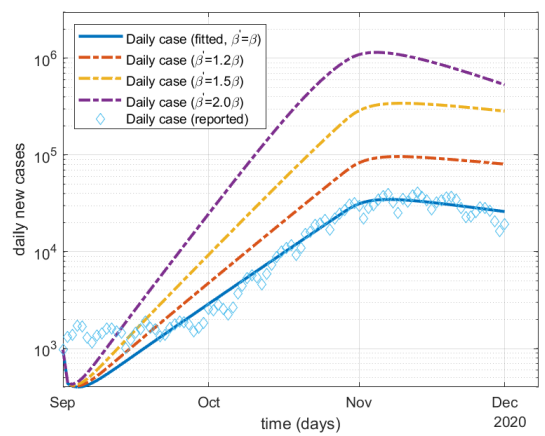

(c) Italy

Figure 16. Simulation of the second wave with more infectious virus mutants but soft interventions (no change to the relaxed interventions in the second wave).

How would the second waves evolve in the next 30 days with more infectious virus mutants? We further estimate the influence of more infectious coronavirus mutants with much higher transmission rates such as the delta and lambda mutants on the trend of the second waves in the three European countries. At the end of December 2020, COVID-19 mutants had been detected in most European countries. For example, the first mutant was reported on 25 December in Germany, 26 December in France, and 23 December in Italy. We simulate the case movement in the next 30 days following the second wave if virus mutants with different levels of transmission largely spread into communities. Figure 17 shows the estimation results corresponding to three scenarios: mutants with $20 \%, 50 \%$ and $100 \%$ infection rate increase, i.e., $\beta^{\prime}=1.2 \beta, \beta^{\prime}=1.5 \beta$, and $\beta^{\prime}=2.0 \beta$. As a result of introducing more infectious viruses, the daily cases would significantly increase, making control even harder and longer. As shown in Table 12, the simulation shows that the mutants with 20\%, 50\% and 100\% extra transmissibility 
Table 12. Change of the estimated daily cases on the $30^{\text {th }}$ day after the second wave caused by more infectious mutants in three European countries, corresponding to Figure 17. $\Delta_{1}$ refers to the change of estimated daily cases on the $30^{\text {th }}$ day compared to the last day of the second wave; $\Delta_{2}$ refers to the change of estimated daily cases compared to the estimated cases at $1.0 \beta$ on the $30^{\text {th }}$ day.

\begin{tabular}{|c|c|c|c|c|c|}
\hline Country & Last Day in $2^{\text {nd }}$ Wave & $1.0 \beta$ & $1.2 \beta\left(\Delta_{1} ; \Delta_{2}\right)$ & $1.5 \beta\left(\Delta_{1} ; \Delta_{2}\right)$ & $2.0 \beta\left(\Delta_{1} ; \Delta_{2}\right)$ \\
\hline Germany & 1,213 & 6,088 & $11,516(\uparrow 849 \% ; \uparrow \uparrow 9 \%)$ & $27,128(\uparrow 2136 \% ; \uparrow 345 \%)$ & $92,825(\uparrow 7552 \% ; \uparrow 1425 \%)$ \\
\hline France & 4,898 & 2,012 & $2,661(\downarrow 45 \% ; \uparrow 32 \%)$ & $3,915(\downarrow 20 \% ; \uparrow 94 \%)$ & $6,962(\uparrow 42 \% ; \uparrow 246 \%)$ \\
\hline Italy & 975 & 16,462 & $19,415(\uparrow 1891 \% ; \uparrow 18 \%)$ & $24,487(\uparrow 2411 \% ; \uparrow 49 \%)$ & $34,858(\uparrow 3475 \% ; \uparrow 112 \%)$ \\
\hline
\end{tabular}

would lead to 11,516 ( 8 times increase compared to the last day of the second wave), 27,128 (21 times increase) and 92,825 (75 times increase) in daily new cases on the $30^{\text {th }}$ day in Germany, building on the same other attributes of the original virus. In France, there would be 2,661 daily new cases (32\% increase over the estimate for the same day without transmissibility increase i.e. at the $1.0 \beta$ infection rate), 3,915 (94\% increase) and 6,962 (246\% increase) compared to an estimate of 19,415 (19 times increase over the last day of the second wave), 24,487 (24 times increase) and 34,858 (35 times increase) in Italy, as a result of increasing the transmission ability through more infectious mutants. As shown in all three countries, if a mutant doubles its transmission rate, then it would change the trend of daily new cases from a downtrend to an upward trend, as shown in Germany (Figure 17(a)) and Italy (Figure 17(c)). Although the simulation results in France look relatively optimistic, they also show the same observation as in Germany and Italy that their second wave would last for much longer with significantly more cases, and it would be much harder to control the spread with their mild interventions. This shows the potential overwhelming outcomes of highly infectious coronavirus mutations in the community no effective control measures are adopted, and vaccination is insufficient.

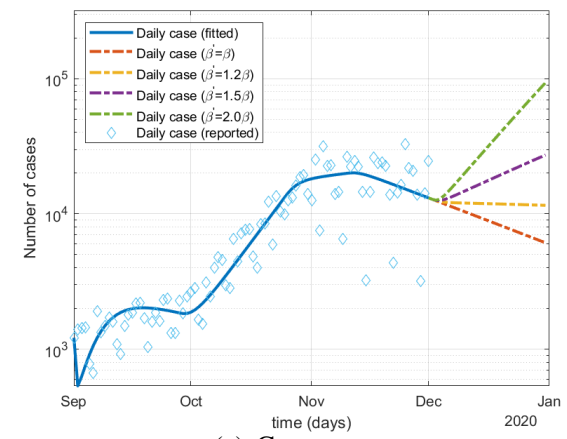

(a) Germany.

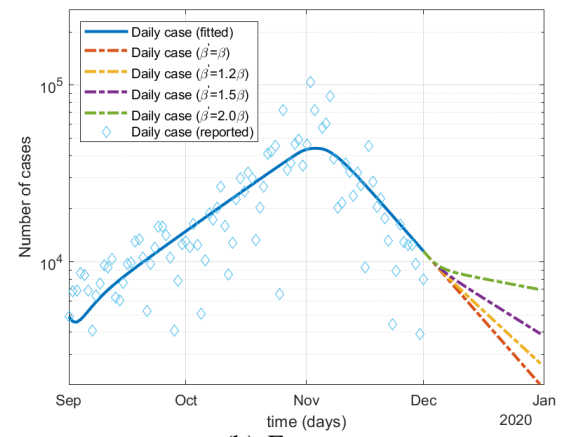

(b) France

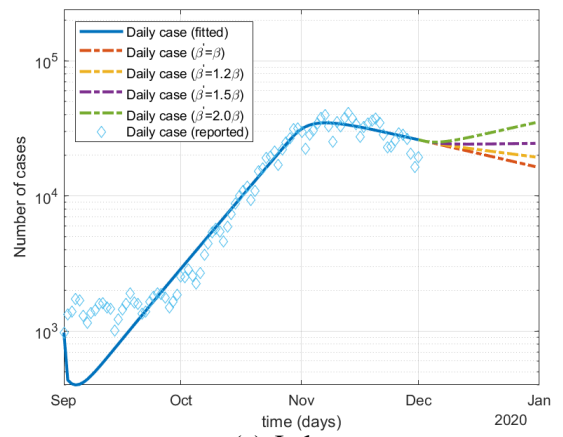

(c) Italy

Figure 17. Estimation of daily cases in the next 30 days following the second waves with more infectious virus mutants.

How well does the model predict the future? We further predict case numbers in the next 10 days following the second wave period, i.e., between 1 September and 1 December, 2020. This test verifies the effectiveness of our model in predicting cases. Since the cases are highly sensitive to the control and relaxation interventions undertaken, it is not appropriate to make a long-range prediction following the existing conditions. Thus we make a prediction for the next 10 days.

The prediction of next 10-day's active, recovered and deceased cases for the three countries is shown in Figure 18. Two metrics, the root mean square error (RMSE) and mean absolute percentage error (MAPE), evaluate the predictive ability of our model. RMSE in Equation (13) is a standard way to measure the error of a model in predicting quantitative data, and MAPE computes the accuracy as a ratio defined by Equation (14). $y_{n}$ is the $n^{\text {th }}$-day's reported value, $\overline{y_{n}}$ is the $n^{\text {th }}$-day's fitted value by our model, and $N$ is the number of observations (in our case $N=10$ ). Since active cases, recovered cases and deaths have different orders of magnitude, the generic percentage of MAPE provides a more intuitive view. The RMSE and MAPE values for the daily active cases, the daily recovered cases and the daily deaths in the three countries are shown in Table 13.

$$
\begin{aligned}
& \text { RMSE }=\sqrt{\frac{1}{N} \sum_{n=1}^{N}\left(y_{n}-\overline{y_{n}}\right)^{2}} \\
& M A P E=\frac{100}{N} \sum_{n=1}^{N} \frac{\left|y_{n}-\overline{y_{n}}\right|}{y_{n}}
\end{aligned}
$$




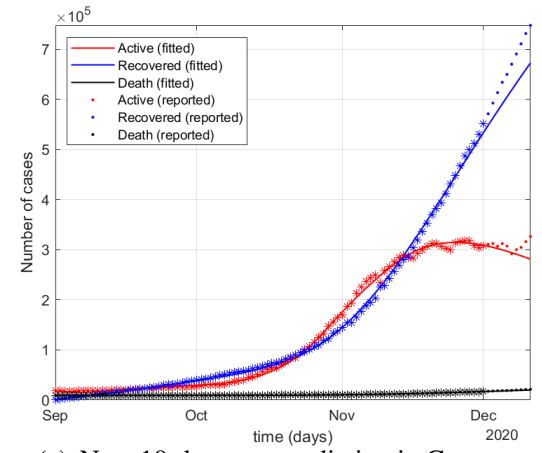

(a) Next 10-day case prediction in Germany.

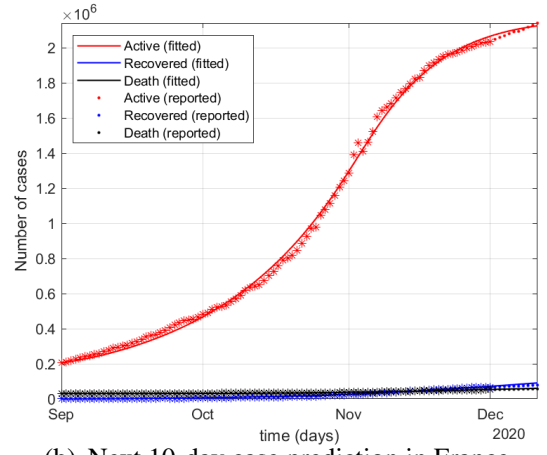

(b) Next 10-day case prediction in France.

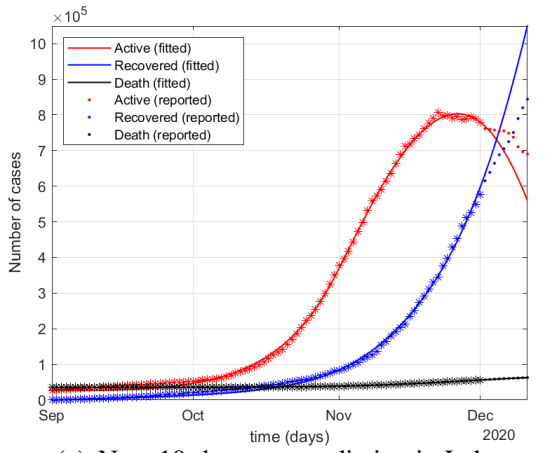

(c) Next 10-day case prediction in Italy.

Figure 18. The prediction of the next 10-day active cases, recovered cases and deaths in three European countries by the SPEIQRD model. The cases during the second wave periods (in star) are the actual, which are used to train the model and infer its parameters. The corresponding lines show the inferred numbers of active, recovered and deceased cases. The actual cases (in dot) of the following 10 days are also shown for comparison.

Table 13. The error of the next 10-day prediction of cases in Germany, France and Italy.

\begin{tabular}{|c|c|c|c|c|c|c|c|c|c|}
\hline \multirow{2}{*}{ Metric } & \multicolumn{3}{|c|}{ Germany } & \multicolumn{3}{c|}{ France } & \multicolumn{3}{c|}{ Italy } \\
\cline { 2 - 10 } & Active & Recovered & Death & Active & Recovered & Death & Active & Recovered & Death \\
\hline RMSE & 19457 & 52652 & 896 & 15927 & 8979 & 2610 & 73434 & 123250 & 280 \\
\hline MAPE & $4.58 \%$ & $7.39 \%$ & $4.12 \%$ & $0.68 \%$ & $11.57 \%$ & $4.51 \%$ & $8.45 \%$ & $14.11 \%$ & $0.40 \%$ \\
\hline
\end{tabular}

Figure 18 shows the predicted active, recovered and death cases of the next 10 days from our model, precluding the overfitting concern. This result shows the model's predictability, as evidenced by Table 13, where RMSE presents the average error of each type of cases, and MAPE computes the normalized error about the prediction. The prediction of active and death cases has good performance in all three countries as the MAPE values are all lower than 10\%, which means that our model can generate robust estimates for the case data. For the recovered cases, the errors are relatively higher. This may be due to more statistical noise in the reported recovered case data than in the death case data. As discussed in $^{3}$, the publicly available case data may contain various quality issues such as inconsistency of testing and reporting, under testing and reporting, missing values, and reporting noises, which also significantly challenge prediction.

\section{Discussion}

This work analyzes the behavior of and difference between the first and second waves and how the different epidemiological dynamics were influenced by control and relaxation interventions under different strategies and with more infectious virus mutants. We characterize, simulate and predict the intrinsic epidemiological attributes of the two waves and the impact of enforcing or relaxing interventions (events) on the two-wave cases by a novel compartmental model SPEIQRD. Our model incorporates both epidemiological and event attributes and their interactions to explicitly simulate the first-to-second wave evolution, the confinement and deconfinement processes, and the future epidemic trend by applying different interventions in the second waves in different European countries. We quantify the influence of control and relaxation events on wave differences with inconsistent case movements. Further, we forecast the possible COVID-19 trends of active, recovered and deceased cases if different control and relaxation interventions are undertaken, to simulate various government responses and control-relaxation intervention combinations. These comprehensive comparisons at four levels - vertical (between waves), horizontal (across countries), what-if (scenario simulations on second waves) and future (30-day trend) - in the two 2020 waves in Germany, France and Italy show that, in the absence of sufficient vaccination, herd immunity, and effective antiviral pharmaceutical treatments, the widespread early or fast relaxation of interventions including public activity restrictions will result in a COVID-19 resurgence. Importantly, we make the first attempt to simulate the impact of virus mutations (such as delta and lambda) with transmissibility increase of 20 to $100 \%$ on the second waves and resurgence infections and the effect of control and relaxation interventions.

In addition to the three countries evaluated, many other countries have also experienced restriction fatigue and resurgences after managing the first wave of COVID-19. Second and multiple waves have been seen not only in Europe but all around the world. Considering the more uncertain and newly emerging factors such as more infectious virus mutants and stranger- 
to-stranger, household and airborne transmissions, a more quantitative and proactive estimation of COVID-19 developments and more timely, effective, targeted and localized restrictions are essential to immediately contain resurgent cases as early as possible and avoid wider and longer regional or national lockdowns and more severe socioeconomic impacts. In balancing epidemic control and societal recovery, policymakers need comprehensive quantitative monitoring and what-if analysis for robust evidence on timely adjusting controls, containing the epidemic, and mitigating societal and psychological impacts.

Our analysis characterizes the changes of epidemiological attributes from the first to second wave. More importantly, our experiments also provide a deep understanding of how and why the COVID-19 pandemic unfolds over waves and the wave time periods by analyzing the interactions between the COVID-19 epidemic and significant external factors. We show how various control or relaxation interventions could affect both resurgence and the impact of virus mutations. This study also informs the following understanding and practice of COVID-19 mitigation by interventions. First, the effectiveness of various non-pharmaceutical interventions directly determines how the virus transmits and the effects. It is recommended more effective control measures are always considered in the initial stage of outbreaks to block transmission and control the outbreak as soon and early as possible. When appropriate, priority should be given to strongly effective policies like full lockdowns, business and school shutdowns, strict social distancing and forbidding gathering as informed by the protection and deconfinement rates of the first waves and our simulations. Interventions should not be eased until the virus has been fundamentally contained. Second, the timing of initiating and maintaining interventions is highly deterministic to the spread period and the infection range, further influencing case movements and other aspects. It is much easier and more efficient to control the virus transmission and prevent wide spread and evolution to a regional or national epidemic in the early stage of outbreaks. Third, the strictness of implementing interventions may affect the lifespan of outbreaks. It would be reasonable to say that, if more strict interventions had been implemented at the very beginning of the second wave in the three countries, their resurgences would have been much weaker and many fewer people would have been infected or died.

However, our study also has several limitations for further exploration. First, our model assumes an intervention is constant over an entire wave, but it may actually be adjusted over time. For example, the strictness of social distancing, such as restrictions on the number of people participating in indoor events, is often adjusted over the epidemic period according to trends in case development, with harsher restrictions applied in more serious conditions. Second, in reality, multiple interventions are often enforced simultaneously, interacting with each other and jointly affecting an epidemic's path. In our modeling, we do not disentangle the impact of multiple events if they were undertaken on the same day since such events are explicitly or implicitly coupled with each other in nature. However, further research could infer the impact of each individual event on case movement for characterization and insight about the positive or negative influence of a specific event. Third, the time periods of the second waves in our case study all end on 1 December 2020, which is just before the confirmation and spread of more transmissible virus mutants. The epidemiological attributes of the virus mutants and their transmission patterns may significantly differ from their original version. Hence, expanding the end date may introduce significant uncertainty and inconsistency to the modeling and findings in this work. However, this treatment also leads to the incompleteness of the second waves in our analysis (for example, in Germany and Italy) and may compromise the accuracy of our results, especially for event-related attributes. Fourth, in simulating the impact of the more infectious virus mutations, we carry forward the epidemiological settings of the original viruses to their mutants, but they need to be adjusted to reflect the epidemiological attributes of specific mutants like delta and lambda. Further, the level and impact of vaccination combined with interventions on mutated virus spread and external factors such as environments and social activities is not yet quantified in terms of epidemiological attributes and mitigation effect. Lastly, the data used may contain various quality issues such as inconsistent, under and missing tests and reporting, small data size, and missing external data such as mobility and environmental factors. For instance, our modeling lacks the degree and extent of actual lockdowns and restrictions and may be biased by the country-based case trends that may significantly vary from the state-based wave behaviors. This could significantly affect modeling performance and results. In addition to enhancing the data quality, modeling low-quality and small COVID case data is a challenging topic for robust forecasting. This requires future research.

\section{References}

1. Leung, K., Wu, J. T., Liu, D. \& Leung, G. M. First-wave COVID-19 transmissibility and severity in China outside Hubei after control measures, and second-wave scenario planning: a modelling impact assessment. The Lancet (2020).

2. Xu, S. \& Li, Y. Beware of the second wave of COVID-19. The Lancet 395, 1321-1322 (2020).

3. Cao, L. \& Liu, Q. COVID-19 modeling: A review. CoRR abs/2104.12556, 1-73 (2021).

4. Cacciapaglia, G., Cot, C. \& Sannino, F. Second wave COVID-19 pandemics in Europe: a temporal playbook. Sci. Reports 10, 1-8 (2020).

5. Faranda, D. \& Alberti, T. Modeling the second wave of COVID-19 infections in france and italy via a stochastic SEIR model. Chaos: An Interdiscip. J. Nonlinear Sci. 30, 1-25 (2020). 
6. Aleta, A. \& Moreno, Y. Age differential analysis of COVID-19 second wave in Europe reveals highest incidence among young adults. medRxiv (2020).

7. Grech, V. \& Cuschieri, S. COVID-19: A global and continental overview of the second wave and its (relatively) attenuated case fatality ratio. Early Hum. Dev. 105211 (2020).

8. Fan, G. et al. Decreased case fatality rate of COVID-19 in the second wave: A study in 53 countries or regions. Transboundary Emerg. Dis. 68, 213-215 (2021).

9. Iftimie, S. et al. First and second waves of coronavirus disease-19: A comparative study in hospitalized patients in Reus, Spain. PLoS ONE 16, 1-13 (2021).

10. Saito, S. et al. First and second COVID-19 waves in Japan: A comparison of disease severity and characteristics: Comparison of the two COVID-19 waves in Japan. The J. Infect. 82, 84-123.

11. Coccia, M. The impact of first and second wave of the COVID-19 pandemic in society: comparative analysis to support control measures to cope with negative effects of future infectious diseases. Environ. Res. 197, 111099 (2021).

12. Ghanbari, B. On forecasting the spread of the COVID-19 in Iran: The second wave. Chaos, Solitons \& Fractals 140, 110176 (2020).

13. Renardy, M., Eisenberg, M. \& Kirschner, D. Predicting the second wave of COVID-19 in Washtenaw County, MI. $J$. Theor. Biol. 507, 110461 (2020).

14. Shim, E., Tariq, A. \& Chowell, G. Spatial variability in reproduction number and doubling time across two waves of the COVID-19 pandemic in South Korea, February to July, 2020. Int. J. Infect. Dis. 102, 1-9 (2021).

15. Rypdal, K., Bianchi, F. M. \& Rypdal, M. Intervention fatigue is the primary cause of strong secondary waves in the COVID-19 pandemic. Int. J. Environ. Res. Public Heal. 17, 1-17 (2020).

16. Lanteri, D., Carco, D., Castorina, P., Ceccarelli, M. \& Cacopardo, B. Containment effort reduction and regrowth patterns of the COVID-19 spreading. Infect. Dis. Model. 6, 632-642 (2021).

17. López, L. \& Rodó, X. The end of social confinement and COVID-19 re-emergence risk. Nat. Hum. Behav. 4, 746-755 (2020).

18. Mahikul, W., Chotsiri, P., Ploddi, K. \& Pan-ngum, W. Evaluating the impact of intervention strategies on the first wave and predicting the second wave of COVID-19 in Thailand: a mathematical modeling study. Biology 10 (2021).

19. Natale, G. D. et al. The evolution of COVID-19 in Italy after the spring of 2020: An unpredicted summer respite followed by a second wave. Int. J. Environ. Res. Public Heal. 17, 1-12 (2020).

20. Zhong, H. \& Wang, W. Mathematical analysis for COVID-19 resurgence in the contaminated environment. Math. Biosci. Eng. 17, 6909-6927 (2020).

21. Huang, B. et al. Integrated vaccination and physical distancing interventions to prevent future COVID-19 waves in Chinese cities. Nat. Hum. Behav. 5, 695-705 (2021).

22. Aleta, A. et al. Modelling the impact of testing, contact tracing and household quarantine on second waves of COVID-19. Nat. Hum. Behav. 4, 964-971 (2020).

23. Ng, V. et al. Projected effects of nonpharmaceutical public health interventions to prevent resurgence of SARS-CoV-2 transmission in Canada. CMAJ 192, E1053-E1064 (2020).

24. Friston, K. J. et al. Second waves, social distancing, and the spread of COVID-19 across the USA. Wellcome Open Res. 5, $1-39(2021)$.

25. Schwarzendahl, F. J., Grauer, J., Liebchen, B. \& Löwen, H. Mutation induced infection waves in diseases like COVID-19. medRxiv 1-10, DOI: 10.1101/2021.07.06.21260067 (2021).

26. Mirri, S., Delnevo, G. \& Roccetti, M. Is a COVID-19 second wave possible in Emilia-Romagna (Italy)? forecasting a future outbreak with particulate pollution and machine learning. Computation 8, 1-25 (2020).

27. Pedro, S. A. et al. Conditions for a second wave of COVID-19 due to interactions between disease dynamics and social processes. Front. Phys. 8, 1-9 (2020).

28. Dong, E., Du, H. \& Gardner, L. An interactive web-based dashboard to track COVID-19 in real time. The Lancet Infect. Dis. 20, 533-534 (2020).

29. Hale, T. et al. A global panel database of pandemic policies (Oxford COVID-19 government response tracker). Nat. Hum. Behav. 5. 
Table 14. List of notations.

\begin{tabular}{|c|l|}
\hline Notation & Statement \\
\hline $\mathrm{S}$ & the susceptible \\
\hline $\mathrm{P}$ & the protected \\
\hline $\mathrm{E}$ & the exposed \\
\hline $\mathrm{I}$ & the infected \\
\hline $\mathrm{Q}$ & the quarantined \\
\hline $\mathrm{R}$ & the recovered \\
\hline $\mathrm{D}$ & the death \\
\hline $\mathrm{N}$ & the population \\
\hline$\alpha$ & the protection rate \\
\hline$\beta$ & the infection rate \\
\hline$\gamma$ & the incubation rate \\
\hline$\delta$ & the quarantine rate \\
\hline$\lambda$ & the recovery rate \\
\hline$\kappa$ & the mortality rate \\
\hline$\tau$ & the deconfinement rate \\
\hline$\pi_{i}^{j}$ & the $j^{\text {th }}$ event in the $i^{\text {th }}$ wave \\
\hline$\nu$ & the time delay \\
\hline
\end{tabular}

30. Peng, L., Yang, W., Zhang, D., Zhuge, C. \& Hong, L. Epidemic analysis of COVID-19 in China by dynamical modeling. arXiv preprint arXiv:2002.06563 (2020).

31. Brooks-Pollock, E. et al. The population attributable fraction (PAF) of cases due to gatherings and groups with relevance to COVID-19 mitigation strategies. Phil. Trans. R. Soc. B 376, 1-5 (2020).

32. López, L. \& Rodo, X. A modified SEIR model to predict the COVID-19 outbreak in Spain and Italy: simulating control scenarios and multi-scale epidemics. Results Phys. 21, 103746 (2021).

33. Chen, Y.-C., Lu, P.-E., Chang, C.-S. \& Liu, T.-H. A time-dependent SIR model for COVID-19 with undetectable infected persons. IEEE Transactions on Netw. Sci. Eng. 7, 3279-3294 (2020).

34. Ambrosio, B. \& Aziz-Alaoui, M. On a coupled time-dependent SIR models fitting with New York and New-Jersey states COVID-19 data. Biology 9, 135 (2020).

\section{Acknowledgements}

This work is sponsored in part by the Australian Research Council Discovery grant (DP190101079) and ARC Future Fellowship grant (FT190100734).

\section{Additional information}

\section{List of notations}

Table 14 lists the notations used in this paper.

Accession codes and data (https://github.com/QingLIU67/waves_comparison).

Competing interests The authors have no financial computing interests. 